\title{
THE LOWER DENSITY CONJECTURE FOR HARMONIC MEASURE
}

\author{
By
}

\author{
SUNHI CHOI
}

\begin{abstract}
In this paper, we establish the lower density conjecture for harmonic measure in simply connected plane domains.
\end{abstract}

\section{Introduction}

Let $\Omega$ be a simply connected planar domain. Harmonic measure $\omega(w, E, \Omega)$ of a set $E$ with respect to a point $w \in \Omega$ can be defined as the normalized linear measure of $f^{-1}(E)$, where $f$ is the Riemann map sending the origin to $w$. Harmonic measure has many applications and connections throughout analysis, probability, and dynamics; and it is of great interest to describe its geomeric properties.

In 1916, F. and M. Riesz [GaMa] proved that if $\partial \Omega$ is rectifiable, then for a set $E \subset \partial \Omega, \omega(E)=0$ iff $\Lambda_{1}(E)=0$ (i.e., $\omega \ll \Lambda_{1} \ll \omega$ ). Makarov [Mak 85] and Pommerenke [Pom 86] improved this result by showing that on the cone points of $\partial \Omega, \omega \ll \Lambda_{1} \ll \omega$ and that the rest of $\partial \Omega$ has a subset with full harmonic measure but with zero length. In 1990, Bishop and Jones [BiJo 90] further generalized the F. and M. Riesz theorem by proving that for every rectifiable curve $\Gamma, \omega \ll \Lambda_{1} \ll \omega$ on $\Gamma \cap \partial \Omega$. In [Bis] and [Bis 91], Bishop stated the lower density conjecture, which would generalize the theorem by Bishop and Jones [BiJo 90], [BiJo 94]. This conjecture is stated later in this section as a corollary of the following result.

Theorem 1. Let

$$
E=\left\{x \in \partial \Omega: \liminf _{r \rightarrow 0} \frac{\omega(B(x, r))}{r}>0\right\} .
$$

Then $\omega \ll \Lambda_{1}$ on $E$.

Theorem 1 has several corollaries. 
Corollary 1. Let $F$ be a subset of $\partial \Omega$ and assume that there exists a constant $M(F)$ such that

$$
\sum \operatorname{rad}\left(B_{i}\right) \leq M(F)<\infty
$$

for every disjoint collection of balls $\left\{B_{i}\right\}$ with center $\left(B_{i}\right) \in F$ and $\operatorname{rad}\left(B_{i}\right) \leq$ $\operatorname{diam}(\partial \Omega)$. Then $\omega \ll \Lambda_{1}$ on $F$.

Proof. Let $F$ be a set satisfying the hypothesis of Corollary 1 with $\Lambda_{1}(F)=0$, then $\omega(F \cap E)=0$ by Theorem 1 . Hence it suffices to prove that

$$
\omega(F \cap(\partial \Omega \backslash E))=0 .
$$

Let $\epsilon>0$. For every $x \in F \cap(\partial \Omega \backslash E)$, there exists $r_{x}>0$ such that

$$
\omega\left(B\left(x, r_{x}\right)\right)<\epsilon r_{x} .
$$

By the basic covering theorem [Mat 95], there also exist disjoint balls $B\left(x_{i}, \frac{1}{5} r_{x_{i}}\right)$ $(i=1,2, \ldots)$ such that

$$
F \cap(\partial \Omega \backslash E) \subset \bigcup_{i} B\left(x_{i}, r_{x_{1}}\right) .
$$

Combining these, we obtain

$$
\omega(F \cap(\partial \Omega \backslash E)) \leq \omega\left(\bigcup_{i} B\left(x_{i}, r_{x_{i}}\right)\right)<5 \epsilon \sum \frac{1}{5} r_{x_{i}}<5 \epsilon M(F)
$$

since $x_{i} \in F$. Hence $\omega(F \cap(\partial \Omega \backslash E))=0$.

Conversely, Theorem 1 can be derived from Corollary 1 , since for $n \in \mathbb{N}$,

$$
E_{n}=\left\{x \in \partial \Omega: \frac{\omega(B(x, r))}{r}>\frac{1}{n}, \text { for every } r>0\right\}
$$

satisfies the hypothesis of Corollary 1 with $M\left(E_{n}\right)=n$.

The next corollary, first proved by Bishop and Jones [BiJo 90], resolves a conjecture of $\emptyset \mathrm{ksendal}[\emptyset \mathrm{ks} 81]$.

Corollary 2. If $\Gamma$ is a rectifiable curve and $\Omega$ is a simply connected domain, then $\omega \ll \Lambda_{1}$ on $\Gamma \cap \partial \Omega$.

Proof. Let $F=\Gamma \cap \partial \Omega$. Then $F$ satisfies the hypothesis of Corollary 1 with $M(F)=\Lambda_{1}(\Gamma)$. Hence $\omega \ll \Lambda_{1}$ on $F$, by Corollary 1 .

The last corollary is the lower density conjecture from [Bis] and [Bis 91]. When $\Omega$ is a simply connected domain, $x \in \partial \Omega$ is called a twist point if both

$$
\liminf _{z \rightarrow x, z \in \Omega} \arg (z-x)=-\infty \text { and } \limsup _{z \rightarrow x, z \in \Omega} \arg (z-x)=+\infty,
$$

where $\arg (z-x)$ denotes a continuous branch of the argument defined in $\Omega$. 
Corollary 3 (the lower density conjecture). At $\omega$-almost every twist point $x \in \partial \Omega$,

$$
\liminf _{r \rightarrow 0} \frac{\omega(B(x, r))}{r}=0 .
$$

Proof. It is known [Mak 85], [Pom 86] that at $\omega$-almost every $x \in \partial \Omega$, either

$$
\text { (a) } \lim _{r \rightarrow 0} \frac{\omega(B(x, r))}{r} \text { exists and } \neq 0, \infty
$$

or

$$
\text { (b) } \limsup _{r \rightarrow 0} \frac{\omega(B(x, r))}{r}=\infty \text {; }
$$

and the set of points satisfying (b) coincides with the set of twist points [Pom 86] except for a set of zero harmonic measure. Hence it suffices to prove

$$
\omega\left(\left\{x \in \partial \Omega: \limsup _{r \rightarrow 0} \frac{\omega(B(x, r))}{r}=\infty, \liminf _{r \rightarrow 0} \frac{\omega(B(x, r))}{r}>0\right\}\right)=0 .
$$

But, by the basic covering theorem,

$$
\Lambda_{1}\left(\left\{x \in \partial \Omega: \limsup _{r \rightarrow 0} \frac{\omega(B(x, r))}{r}=\infty\right\}\right)=0 .
$$

Hence (1.1) follows from Theorem 1.

The rest of this paper is devoted to proving Theorem 1. A general sketch of the proof is as follows. First, we fix a lower bound $a>0$ and a scale $R>0$ and let

$$
A_{a, R}=\left\{x \in \partial \Omega: a<\liminf _{r \rightarrow 0} \frac{\omega(B(x, r))}{r}<(1.01) a, a<\frac{\omega(B(x, r))}{r}, \forall r \leq R\right\} .
$$

Given $x \in A_{a, R}$ and a radius $r(r<R)$ for which the liminf is almost attained, it is shown that if $B\left(x_{i}, r_{i}\right)$ are disjoint disks in $B(x, r)$ centered at points of $A_{a, R}$, then

$$
\sum r_{i} \leq \frac{1}{a} \sum \omega\left(B\left(x_{i}, r_{i}\right)\right) \leq \frac{1}{a} \omega(B(x, r)) \leq(1.01) r
$$

If Theorem 1 failed, there would exist $x$ in some $A_{a, R}$ and a small radius $r$ chosen as above such that $\omega\left(B(x, r) \cap A_{a, R}\right)$ is close to $\omega(B(x, r))$ and $\Lambda_{1}\left(B(x, r) \cap A_{a, R}\right)=0$. Assuming this, we construct a Lipschitz graph in $B(x, r)$ with desirable properties. Since the length of $B(x, r) \cap A_{a, R}$ is zero, we can choose small intervals $I_{i}$ intersecting $A_{a, R}$ and a Lipschitz graph connecting them such that the graph has big spikes between $I_{i}$ and all points of $A_{a, R}$ are located on or above the graph. We observe that most of the points of $\partial \Omega$ are located on or above the Lipschitz graph since $A_{a, R}$ has almost full harmonic measure in $B(x, r)$. Also, 
$I_{i}$ can be chosen to have points of $A_{a, R}$ in every ring domain surrounding $\left\{I_{i}\right\}$ and with some fixed small modulus. Since the length of our Lipschitz graph is a large multiple of $r$, by suitably drawing ring domains, we can construct a disjoint collection of disks in $B(x, r)$ centered at $A_{a, R}$ the sum of whose radii is greater than (1.01)r. This gives the desired contradiction.

\section{Preliminary reductions}

In this section, Theorem 1 is reduced to a simpler formulation.

We first reduce the domain to a Jordan domain. Since Theorem 1 is equivalent to Corollary 1 , it suffices to prove that if Corollary 1 holds for every Jordan domain, then it also holds for every simply connected domain $\Omega$. For the proof, let $F$ be a subset of $\partial \Omega$ satisfying the hypothesis of Corollary 1 and let

$$
\Lambda_{1}(F)=0 .
$$

By the Moore triod theorem [Pom 91], at $\omega$-almost every $x \in \partial \Omega$,

$$
\operatorname{card}\left\{f^{-1}(x)\right\} \leq 2 .
$$

Hence $f$ is at most two-to-one on a subset $E$ of $f^{-1}(F)$ with full measure. Let $T$ be the family of the shorter arcs on $\partial \mathbb{D}$ which connect a pair of identified points in $E$. Define

$$
T_{i}=\{I \in T: 1 /(i+1) \leq|I|<1 / i\}
$$

and let $E_{i}^{l}$ (resp. $E_{i}^{r}$ ) be the set of the left (resp. right) endpoints of the intervals in $T_{i}$. Note that $f$ is one-to-one on a set which does not intersect $E_{i}^{l}$ (or $E_{i}^{r}$ ).

Fix $i$. Each interval $I \in T_{i}$ has a small subinterval $I^{\prime}$ with common left endpoint such that $I^{\prime} \cap\left(\bigcup_{k \leq i} E_{k}^{r}\right)=\emptyset$, since for any interval $J$ with identified endpoints, either $I \subset J$ or $J \subset I$. Let

$$
E_{1}=\bigcup_{i}\left(\bigcup_{I \in T_{i}} I^{\prime} \backslash \bigcup_{\substack{I \in T_{k} \\ k \geq i+1}} I\right) \bigcap E
$$

and let $E_{2}=E \backslash E_{1}$. Since $\bigcup_{i} E_{i}^{l} \subset E_{1}$ and $\bigcup_{i} E_{i}^{r} \cap E_{1}=\emptyset, f$ is one-to-one on both $E_{1}$ and $E_{2}$. Since $E_{i}(i=1,2)$ are measurable, $f\left(\bigcup_{\zeta \in E_{i}} \Gamma_{1 / 2}(\zeta)\right)$ are Jordan domains, where

$$
\Gamma_{\frac{1}{2}}(\zeta)=\left\{z \in \mathbb{D}: 1-|z|>\frac{1}{2}|\zeta-z|\right\} .
$$

Since $\Lambda_{1}\left(f\left(E_{i}\right)\right)=\Lambda_{1}(F)=0$, Corollary 1 on Jordan domains implies $\omega\left(f\left(E_{i}\right)\right)=0$ and hence $2 \pi \omega(F)=\Lambda_{1}(E)=\Lambda_{1}\left(E_{1}\right)+\Lambda_{1}\left(E_{2}\right)=0$. 
Next, we observe that the set $E$ of Theorem 1 is a countable union of sets of the form $A_{a, R}$ defined below. Hence Theorem 1 can be reduced to the following theorem.

Theorem 2. Let $\Omega$ be a Jordan domain and let $\omega(\cdot)=\omega\left(w_{0}, \cdot, \Omega\right)$ for $w_{0} \in \Omega$. Given $a>0$ and $R>0$, define

$$
A_{a, R}=\left\{x \in \partial \Omega: a<\liminf _{r \rightarrow 0} \frac{\omega(B(x, r))}{r}<(1.01) a, a<\frac{\omega(B(x, r))}{r}, \forall r \leq R\right\} .
$$

Then $\omega \ll \Lambda_{1}$ on $A_{a, R}$.

For the proof of Theorem 2, we first let $f$ be a conformal mapping from $\mathbb{D}$ to $\Omega$. If Theorem 2 failed, we could fix $a>0$ and $R>0$ such that for some subset $A$ of $f^{-1}\left(A_{a, R}\right), \Lambda_{1}(f(A))=0$ but $\omega(f(A))>0$. Assuming this, we also fix a density point $\zeta_{0}$ of $A$ throughout Sections 3-6. The contradiction derived in Section 6 proves Theorem 2 .

\section{Local behavior of $\partial \Omega$ near $f\left(\zeta_{0}\right)$}

In Lemma 3.1, we describe the behavior of $\partial \Omega$ in a neighborhood of $f\left(\zeta_{0}\right)$. Lemmas 3.2, 3.3 and 3.4 are steps for proving Lemma 3.1 and are not used in the other sections. Hence this section can be read independently of the rest of this paper.

Let $\operatorname{dist}_{\Omega}(A, B)$ denote the length of the shortest path in $\Omega$ connecting a point of $A$ to a point of $B$.

Lemma 3.1. For any $t(0<t<1)$, there exists an arc $I$ on $\partial \mathbb{D}$ containing $\zeta_{0}$ such that for some $r_{0}<R$, the following hold.

(i) For every arc $J$ such that $\zeta_{0} \in J \subset I,|J \cap A| /|J| \geq t$.

(ii) Let $\beta=f(I)$ and $x=f\left(\zeta_{0}\right)$. Then the endpoints of $\beta$ lie on $\partial B\left(x, r_{0}\right)$, and there exists a circular crosscut on $\partial B\left(x, r_{0}\right) \cap \Omega$ separating $\beta$ from $w_{0}$.

(iii) Let $\left\{B_{i}\right\}$ be any disjoint collection of balls in $B\left(x, r_{0}\right)$ with centers in $f(A)$. Then

$$
\sum \operatorname{rad}\left(B_{i}\right) \leq(1.01) r_{0}
$$

(iv) Let $\beta_{l}$ and $\beta_{r}$ be the left and right subarcs of $\beta$ with one endpoint at $x$. Then

$$
\left.\operatorname{dist}_{\Omega \cap B\left(x, \frac{99}{100}\right.} r_{0}\right) \backslash B\left(x, \frac{1}{10} r_{0}\right)\left(\beta_{l}, \beta_{r}\right) \approx r_{0}
$$


(v) There exists a point $f(z)$ in $\Omega \cap B\left(f\left(\zeta_{0}\right), \frac{2}{5} r_{0}\right)$ such that for some $r \approx r_{0}$

$$
B(f(z), r) \subset \Omega \cap B\left(f\left(\zeta_{0}\right), r_{0}\right)
$$

and

$$
\omega\left(w_{0}, \partial B\left(f(z), \frac{1}{2} r\right), \Omega \backslash B\left(f(z), \frac{1}{2} r\right)\right) \approx \omega(\beta)
$$

The proof of Lemma 3.1 is given at the end of this section.

In the following lemma, we relate extremal length to harmonic measure. Let $x \in \partial \Omega$ and let $s$ and $r$ be small numbers such that $0<s<r e^{-4 \pi}$. Let $\beta$ be an arc on $\partial \Omega$ containing $x$ such that there exists an arc $J \subset \partial B(x, r) \cap \Omega$ which separates $\beta$ from $w_{0}$ and which has the same endpoints as $\beta$. Let $\Omega_{\beta}$ be the subregion of $\Omega$ bounded by $\beta$ and $J$, and let $x_{0}$ and $x_{1}$ be the points in $\beta \cap \partial B(x, s)$ such that the subarcs of $\beta$ connecting the endpoints of $\beta$ and $x_{i}$ do not intersect $\overline{B(x, s)}$. (See Figure 1.)

Lemma 3.2. Let $\Omega^{\prime}$ be the region obtained by replacing $\beta_{\left(x_{0}, x_{1}\right)}$ with $(\partial B(x, s))_{\left(x_{0}, x_{1}\right)}$, where $\beta_{\left(x_{0}, x_{1}\right)}$ is the subarc of $\beta$ connecting $x_{0}$ and $x_{1}$ via $x$ and $(\partial B(x, s))_{\left(x_{0}, x_{1}\right)}$ is the subarc of $\partial B(x, s)$ connecting $x_{0}$ and $x_{1}$ such that $\partial \Omega^{\prime}$ separates $w_{0}$ from $x$. Let $\Gamma$ be the path family in $(B(x, r) \backslash B(x, s)) \cap \Omega^{\prime}$ connecting $\bar{\Omega}_{\beta} \cap \partial B(x, r)$ and $(\partial B(x, s))_{\left(x_{0}, x_{1}\right)}$, and let

$$
\lambda(\Gamma)=\frac{m}{\pi} \log \frac{r}{s},
$$

where $\lambda$ denotes extremal length. Then for some $C>0$ independent of $m$,

$$
\frac{\omega(\beta \cap B(x, s))}{s} \leq C \frac{\omega(\beta)}{r}\left(\frac{s}{r}\right)^{m-1} .
$$

Proof. Let $x_{2}, x_{3}$ be the endpoints of $J$ and let $\Omega_{\beta}^{\prime}$ be the region bounded by $\beta_{\left(x_{0}, x_{2}\right)}, \beta_{\left(x_{1}, x_{3}\right)}, J$, and $(\partial B(x, s))_{\left(x_{0}, x_{1}\right)}$. (See Figure 1.) For the path family $\Gamma^{\prime}$ in $\Omega_{\beta}^{\prime}$ connecting $J$ and $(\partial B(x, s))_{\left(x_{0}, x_{1}\right)}$, let

$$
\lambda\left(\Gamma^{\prime}\right)=\frac{m^{\prime}}{\pi} \log \frac{r}{s} .
$$

Then we obtain

$$
\frac{m}{\pi} \log \frac{r}{s}=\lambda(\Gamma) \leq \lambda\left(\Gamma^{\prime}\right)=\frac{m^{\prime}}{\pi} \log \frac{r}{s}
$$

by the extension rule of extremal length [GaMa], [Pom 91]. Let $\phi$ be a conformal map from $\Omega_{\beta}^{\prime}$ to a rectangle such that $J$ and $(\partial B(x, s))_{\left(x_{0}, x_{1}\right)}$ are mapped to vertical line segments with length $\pi / m^{\prime}$ and $\beta_{\left(x_{0}, x_{2}\right)}$ and $\beta_{\left(x_{1}, x_{s}\right)}$ are mapped to horizontal 


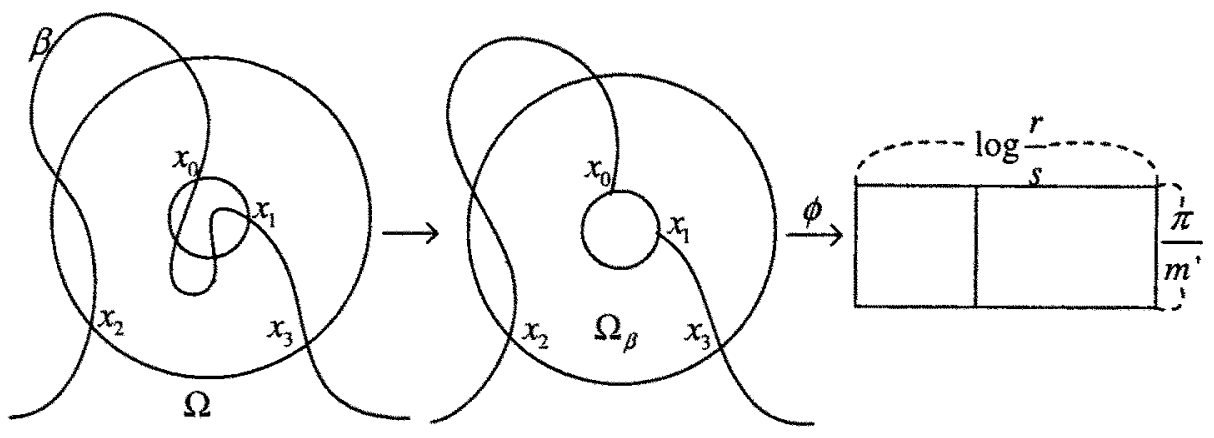

Figure 1. Lemma 3.2.

line segments with length $\log (r / s)$. Let $L$ be the vertical crosscut of this rectangle such that

$$
\operatorname{dist}(\phi(J), L)=\pi / m^{\prime} .
$$

(Such a segment exists in the left half of the rectangle, since $s$ was assumed to be smaller that $r e^{-4 \pi}$ and $m^{\prime} \geq m>\frac{1}{2}$, where the right-hand inequality comes from the extension rule of extremal length for the annulus.)

Let $\Gamma^{\prime \prime}$ be the path family from $L$ to $\phi\left((\partial B(x, s))_{\left(x_{0}, x_{1}\right)}\right)$. Then for some $C>0$,

$$
\begin{aligned}
\omega(\beta) & \geq \omega\left(w_{0}, \beta_{\left(x_{0}, x_{2}\right)} \cup \beta_{\left(x_{1}, x_{3}\right)}, \Omega^{\prime}\right) \\
& \geq \omega\left(w_{0}, \phi^{-1}(L), \Omega^{\prime \prime}\right) \min _{y \in L} \omega\left(y, \phi\left(\beta_{\left(x_{0}, x_{2}\right)} \cup \beta_{\left(x_{1}, x_{3}\right)}\right), \text { Range }(\phi)\right) \\
& \geq C \omega\left(w_{0}, \phi^{-1}(L), \Omega^{\prime \prime}\right)
\end{aligned}
$$

where $\Omega^{\prime \prime}$ is the component of $\Omega^{\prime} \backslash \phi^{-1}(L)$ containing $w_{0}$, and the final inequality uses that $L$ is bounded away from the right-hand edge of the rectangle. Hence

$$
\begin{array}{r}
\omega(\beta \cap B(x, s)) \leq \omega\left(w_{0}, \phi^{-1}(L), \Omega^{\prime \prime}\right) \max _{y \in L} \omega\left(y, \phi\left((\partial B(x, s))_{\left(x_{0}, x_{1}\right)}\right), \text { Range }(\phi)\right) \\
\leq \frac{8}{\pi} \omega\left(w_{0}, \phi^{-1}(L), \Omega^{\prime \prime}\right) e^{-\pi \lambda\left(\Gamma^{\prime \prime}\right)} \leq C \omega(\beta)\left(\frac{s}{r}\right)^{m^{\prime}} \leq C \omega(\beta)\left(\frac{s}{r}\right)^{m},
\end{array}
$$

where the second inequality follows from Beurling's theorem about extremal distance and harmonic measure [GaMa], [Pom 91] and the last inequality follows from (3.2). This implies

$$
\frac{\omega(\beta \cap B(x, s))}{s} \leq C \frac{\omega(\beta)}{r}\left(\frac{s}{r}\right)^{m-1} .
$$


Lemma 3.3. Let $y_{i}(i=1,2, \ldots)$ and $b$ be positive numbers such that

$$
\sum_{i} \frac{1}{y_{i}}=1 \text { and } 0<b<e^{-2} .
$$

Then

$$
\sum_{i} b^{y_{i}} \leq b
$$

Proof. For $b<1$, let $f(x)=b^{1 / x}$. Then $f^{\prime \prime}(x)=b^{1 / x} x^{-4} \log b(\log b+2 x)$, which is positive on $(0,1)$ if $b<e^{-2}$. Then for $0<x<a<1$, we have $f^{\prime}(x)$ is increasing, so $(f(x)+f(a-x))^{\prime}$ is positive if $x>a / 2$ and negative if $x<a / 2$. Thus $b^{1 / x}+b^{1 /(a-x)}$ takes its maximum when $x=0$ and $x=a$. In the lemma, we can replace any pair $y_{j}, y_{k}$ such that $1 / y_{j}+1 / y_{k}=a$ by $y_{j}=\infty$ and $y_{k}=1 / a$ while increasing the sum $\sum_{i} b^{y_{i}}$. The proof is completed by induction.

In Lemma 3.4, we let $\beta$ be an arc on $\partial \Omega$ such that $\beta$ contains $x$ and has a circular crosscut on $\partial B(x, r) \cap \Omega$ separating it from $w_{0}$.

Lemma 3.4. Assume $\omega(B(x, s)) / s>a$, for every s such that $0<s \leq r$. There exists $C<\infty$ such that for all sufficiently small $\epsilon_{1}>0$,

$$
\frac{\omega(\beta \cap B(x, r))}{r} \leq \epsilon_{1} a
$$

implies that there exists an arc $\beta^{\prime}$ on $\partial \Omega$ such that $\beta^{\prime}$ has a circular crosscut on $\partial B(x, r) \cap \Omega$ separating $\beta^{\prime}$ from $w_{0}$ and

$$
a^{2} \leq C \frac{\omega(\beta)}{r} \frac{\omega\left(\beta^{\prime}\right)}{r}
$$

Proof. Since $\partial \Omega$ is a Jordan curve,

$$
\frac{\omega(\beta \cap B(x, s))}{s}=\frac{\omega(B(x, s))}{s}>a
$$

for sufficiently small $s>0$. Hence we can define

$$
s_{0}=\sup \left\{s<r: \frac{\omega(\beta \cap B(x, s))}{s} \geq \frac{a}{2}\right\}>0 .
$$

Since $\omega(\beta \cap B(x, r))$ is increasing in $r$, the only discontinuities it can have are upwards jump discontinuities; hence

$$
\frac{\omega\left(\beta \cap B\left(x, s_{0}\right)\right)}{s_{0}}=\frac{a}{2} .
$$

Also, if $\epsilon_{1}<e^{-4 \pi} / 2$,

$$
s_{0} / r<e^{-4 \pi}
$$


Let $\left\{\beta_{i}\right\}$ be the disjoint collection of arcs on $\partial \Omega$ having a circular crosscut $J_{i}$ on $\partial B(x, r) \cap \Omega$ and satisfying $\beta_{i} \cap B\left(x, s_{0}\right) \neq \emptyset$. Denote $\beta_{0}=\beta$, and let $x_{0}^{i}$ and $x_{1}^{i}$ be the points on $\beta_{i} \cap \partial B\left(x, s_{0}\right)$ through which $\beta_{i}$ hits $\partial B\left(x, s_{0}\right)$ first from the endpoints of $\beta_{i}$. Let $\Gamma_{i}$ be the path family in $\Omega_{\beta_{i}}^{\prime}$ connecting $\bar{\Omega}_{\beta_{i}} \cap \partial B(x, r)$ and $\left(\partial B\left(x, s_{0}\right)\right)_{\left(x_{0}^{i}, x_{1}^{i}\right)}$, and let

$$
\lambda\left(\Gamma_{i}\right)=\frac{m_{i}}{\pi} \log \frac{r}{s_{0}} .
$$

Then

$$
\begin{aligned}
\frac{a}{2}=a-\frac{\omega\left(\beta_{0} \cap B\left(x, s_{0}\right)\right)}{s_{0}} & \leq \sum_{i=1,2 . .} \frac{\omega\left(\beta_{i} \cap B\left(x, s_{0}\right)\right)}{s_{0}} \\
& \leq C \sum_{i=1,2 . .} \frac{\omega\left(\beta_{i}\right)}{r}\left(\frac{s_{0}}{r}\right)^{m_{i}-1} \\
& \leq C \max _{i=1,2 . .}\left\{\frac{\omega\left(\beta_{i}\right)}{r}\right\} \sum_{i=1,2 . .}\left(\frac{s_{0}}{r}\right)^{m_{i}-1}
\end{aligned}
$$

where the first equality follows from the definition of $s_{0}$ and the second inequality follows from Lemma 3.2.

On the other hand, Lemma 3.2 implies

$$
\frac{a}{2}=\frac{\omega\left(\beta_{0} \cap B\left(x, s_{0}\right)\right)}{s_{0}} \leq C \frac{\omega\left(\beta_{0}\right)}{r}\left(\frac{s_{0}}{r}\right)^{m_{0}-1} .
$$

If $\epsilon_{1}<1 / 4 C$, where $C$ is the constant from (3.4), then the right-hand side of (3.4) is smaller than $\frac{1}{4} a\left(s_{0} / r\right)^{m_{0}-1}$. Since $s_{0} / r<e^{-4 \pi}$,

$$
\frac{1}{2}<m_{0}<1
$$

where the left- and right-hand inequalities follow from the extremal length estimate for the annulus and (3.4), respectively. Let $m^{\prime}$ be the number satisfying

$$
\frac{1}{m^{\prime}}=\sum_{i=1,2, \ldots} \frac{1}{m_{i}}
$$

Then by the parallel rule of extremal length [GaMa], [Pom 91] and (3.5),

$$
\frac{1}{m^{\prime}} \leq 2-\frac{1}{m_{0}}<1
$$

This implies $m^{\prime}>1$ and

$$
1-m_{0} \leq m^{\prime}-1
$$

Define $y_{i}=m_{i} / m^{\prime} ;$ then

$$
\sum_{i=1,2, \ldots} \frac{1}{y_{i}}=\sum_{i=1,2, \ldots} \frac{m^{\prime}}{m_{i}}=1 .
$$


Also, we have

$$
\left(\frac{s_{0}}{r}\right)^{m^{\prime}}<\left(e^{-4 \pi}\right)^{m^{\prime}}<e^{-4 \pi}
$$

since $m^{\prime}>1$. Thus by Lemma 3.3,

$$
\sum_{i=1,2 . .}\left(\frac{s_{0}}{r}\right)^{m_{i}}=\sum_{i=1,2 . .}\left(\left(\frac{s_{0}}{r}\right)^{m^{\prime}}\right)^{y_{i}} \leq\left(\frac{s_{0}}{r}\right)^{m^{\prime}} .
$$

Now (3.3), (3.4), (3.7) and (3.8) show that

$$
\begin{array}{r}
\frac{a}{2} \leq C \max _{i=1,2, \ldots}\left\{\frac{\omega\left(\beta_{i}\right)}{r}\right\} \sum_{i=1,2, \ldots}\left(\frac{s_{0}}{r}\right)^{m_{i}-1} \leq C \max _{i=1,2, \ldots}\left\{\frac{\omega\left(\beta_{i}\right)}{r}\right\}\left(\frac{s_{0}}{r}\right)^{m^{\prime}-1} \\
\leq C \max _{i=1,2, \ldots}\left\{\frac{\omega\left(\beta_{i}\right)}{r}\right\}\left(\frac{s_{0}}{r}\right)^{1-m_{0}} \leq C^{2} \max _{i=1,2, \ldots}\left\{\frac{\omega\left(\beta_{i}\right)}{r}\right\} \frac{\omega\left(\beta_{0}\right)}{r} \frac{2}{a} .
\end{array}
$$

Hence there exists $i$ such that

$$
a^{2} \leq C \frac{\omega\left(\beta_{0}\right)}{r} \frac{\omega\left(\beta_{i}\right)}{r}
$$

for some $C>0$.

Now we prove Lemma 3.1, using Lemma 3.4.

Proof. Since $\zeta_{0}$ is a point of density of $A$, there exists a sufficiently small arc $I$ on $\partial \mathbb{D}$ satisfying (i), (ii) and the inequality

$$
a<\frac{\omega\left(B\left(x, r_{0}\right)\right)}{r_{0}}<(1.01) a
$$

Let $\left\{B_{i}\right\}$ be a disjoint collection of balls in $B\left(x, r_{0}\right)$ with centers in $f(A)$; then

$$
\sum_{i} a \cdot \operatorname{rad}\left(B_{i}\right)<\sum_{i} \omega\left(B_{i}\right) \leq \omega\left(B\left(x, r_{0}\right)\right)<(1.01) a r_{0},
$$

where the left inequality follows from the definition of $A$. This implies (iii).

To prove (iv), let $\frac{1}{10} r_{0} \leq s \leq r_{0}$ and let $\beta_{s}$ be the subarc of $\beta$ containing $x$ and having a circular crosscut on $\partial B(x, s) \cap \Omega$. Let $\epsilon_{2}>0$ be a constant smaller than $\epsilon_{1}$ and satisfying $20 C \epsilon_{2}<1$, where $\epsilon_{1}$ and $C$ are the constants from Lemma 3.4. Then $\omega\left(\beta_{s}\right) / s \leq \epsilon_{2} a$ implies that there exists an arc $\beta_{s}^{\prime}$ such that

$$
a^{2} \leq C \frac{\omega\left(\beta_{s}\right)}{s} \frac{\omega\left(\beta_{s}^{\prime}\right)}{s} \leq C \epsilon_{2} a \frac{\omega\left(\beta_{s}^{\prime}\right)}{s} \leq \frac{a}{2} \frac{\omega\left(B\left(x, r_{0}\right)\right)}{r_{0}} .
$$

This contradicts (3.9), and hence we obtain $\omega\left(\beta_{s}\right) / s \approx a$ for every $s \in\left[\frac{1}{10} r_{0}, r_{0}\right]$.

On the other hand, let $\alpha$ and $\beta$ be arcs on $\partial \Omega$ with $\alpha \subset \beta$, and let $x_{1}, x_{2}$ be points of distinct components of $\beta \backslash \alpha$ with $\operatorname{dist}\left(\alpha, x_{i}\right) \geq 1$. Given any $\epsilon>0$, there exists 
$\delta>0$ such that $\left|x_{1}-x_{2}\right| \leq \delta$ implies $\omega(z, \alpha, \Omega) \leq \epsilon(z, \beta, \Omega)$ for every $z \in \Omega$ with $\omega(z, \beta, \Omega)>\frac{1}{2}$. This proves (iv), since $\omega\left(\beta_{s}\right) / s \approx a$ for every $s \in\left[\frac{1}{10} r_{0}, r_{0}\right]$.

By (iv), there exists a point $f(z)$ in $\Omega \cap B\left(f\left(\zeta_{0}\right), \frac{2}{5} r_{0}\right)$ satisfying (3.1) with $r \approx r_{0}$. Also, (iv) combined with the extremal length estimate for $\Omega \cap\left(B\left(f\left(\zeta_{0}\right), \frac{99}{100} r_{0}\right) \backslash B\left(f\left(\zeta_{0}\right), \frac{1}{10} r_{0}\right)\right)$ shows that

$$
\omega\left(w_{0}, \partial B\left(f(z), \frac{1}{2} r\right), \Omega \backslash B\left(f(z), \frac{1}{2} r\right)\right)
$$

is bounded below by a small multiple of $\omega(\beta)$. Hence $(v)$ is proved.

\section{Three standard lemmas}

In this section, we recall three lemmas relating harmonic measure to the geometry of a domain. They will be frequently used in Sections 5 and 6.

The following lemma is a refinement of a theorem in [McM 70].

Lemma 4.1. Let $\Omega$ be a Jordan domain and $\omega(\cdot)=\omega\left(w_{0}, \cdot, \Omega\right)$ for $w_{0} \in \Omega$. Let $d>0$ and $R_{1}, R_{2}$ be ring domains such that

$$
\bmod \left(R_{1}\right), \bmod \left(R_{2}\right) \geq d
$$

and the inner curve of $\partial R_{1}$ coincides with the outer curve of $\partial R_{2}$. Let $\gamma^{1}, \gamma^{2}$ denote the outer and inner curves of $\partial R_{1}$, respectively, and let $\gamma^{3}$ denote the inner curve of $\partial R_{2}$. For $\zeta_{0}, \zeta_{1} \in \gamma^{i}$, we also let $\gamma_{\left(\zeta_{0}, \zeta_{1}\right)}^{i}$ denote a subarc of $\gamma^{i}$ connecting $\zeta_{0}$ and $\zeta_{1}$. Suppose that the arc $\beta \subset \partial \Omega$ satisfies the following conditions:

(i) $\zeta_{0}, \zeta_{1} \in \gamma^{2}$ where $\zeta_{0}$ and $\zeta_{1}$ are the endpoints of $\beta$;

(ii) $\gamma_{\left(\zeta_{0}, \zeta_{1}\right)}^{2} \subset \Omega$;

(iii) $\gamma_{\left(\zeta_{0}, \zeta_{1}\right)}^{2}$ separates $\beta$ from $w_{0}$;

(iv) $\gamma^{3} \cap \beta \neq \emptyset$.

Then for some constant $c(d)$ depending on $d$,

$$
\frac{\omega\left(\beta \cap\left(R_{1} \cup R_{2}\right)\right)}{\omega(\beta)} \geq c(d)>0 .
$$

Note that $c(d) \rightarrow 0$, as $d \rightarrow 0$.

Proof. Let $\zeta_{2}, \zeta_{3}$ be the points on $\beta$ satisfying

$$
\zeta_{2}, \zeta_{3} \in \beta \cap \gamma_{3} \quad \text { and } \quad\left(\beta_{\left(\zeta_{0}, \zeta_{2}\right)} \cup \beta_{\left(\zeta_{1}, \zeta_{3}\right)}\right) \cap \gamma_{3}=\emptyset
$$

(See Figure 2.) We show that for some constant $c(d)$ depending on $d$,

$$
\omega\left(\beta_{\left(\zeta_{2}, \zeta_{3}\right)} \cap\left(R_{1} \cup R_{2}\right)^{c}\right) \leq c(d) \omega\left(\beta_{\left(\zeta_{0}, \zeta_{2}\right)} \cup \beta_{\left(\zeta_{1}, \zeta_{3}\right)}\right) .
$$




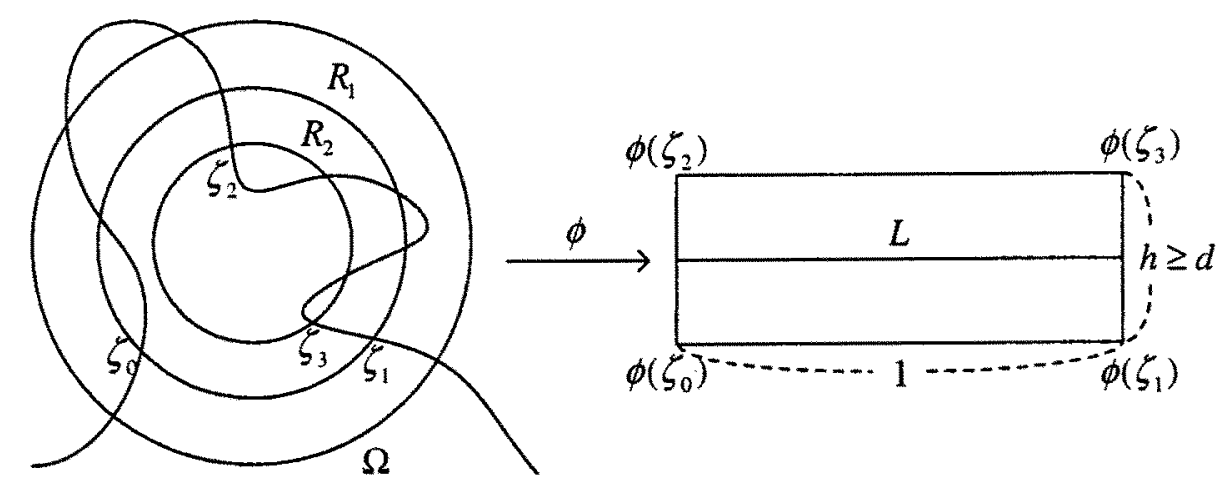

Figure 2. Lemma 4.1.

Let $\phi$ be a conformal map sending the region bounded by $\gamma_{\left(\zeta_{0}, \zeta_{1}\right)}^{2}, \gamma_{\left(\zeta_{2}, \zeta_{3}\right)}^{3}, \beta_{\left(\zeta_{0}, \zeta_{2}\right)}$ and $\beta_{\left(\zeta_{1}, \zeta_{3}\right)}$ to a rectangle. Then by the extension rule of extremal length [GaMa], [Pom 91],

$$
\frac{\left|\phi\left(\zeta_{3}\right)-\phi\left(\zeta_{1}\right)\right|}{\left|\phi\left(\zeta_{0}\right)-\phi\left(\zeta_{1}\right)\right|} \geq \bmod \left(R_{2}\right) \geq d
$$

Let $L$ be the line segment bisecting $\phi\left(\beta_{\left(\zeta_{0}, \zeta_{2}\right)}\right)$ and $\phi\left(\beta_{\left(\zeta_{1}, \zeta_{3}\right)}\right)$. Then

$$
\begin{array}{r}
\omega\left(\beta_{\left(\zeta_{0}, \zeta_{2}\right)} \cup \beta_{\left(\zeta_{1}, \zeta_{3}\right)}\right) \geq \omega\left(w_{0}, \phi^{-1}(L), \Omega^{\prime}\right) \min _{x \in L} \omega\left(x, \phi\left(\beta_{\left(\zeta_{0}, \zeta_{2}\right)} \cup \beta_{\left(\zeta_{1}, \zeta_{3}\right)}\right), \text { range }(\phi)\right) \\
\geq c(d) \omega\left(w_{0}, \phi^{-1}(L), \Omega^{\prime}\right)
\end{array}
$$

and

$$
\omega\left(w_{0}, \phi^{-1}(L), \Omega^{\prime}\right) \geq \omega\left(w_{0}, \gamma_{\left(\zeta_{2}, \zeta_{3}\right)}^{3}, \Omega^{\prime \prime}\right) \geq \omega\left(\beta_{\left(\zeta_{2}, \zeta_{3}\right)} \cap\left(R_{1} \cup R_{2}\right)^{c}\right),
$$

where $\Omega^{\prime}$ and $\Omega^{\prime \prime}$ are the regions obtained by replacing the subarc of $\beta$ connecting two endpoints of $\phi^{-1}(L)$ and $\gamma_{\left(\zeta_{2}, \zeta_{3}\right)}^{3}$ with $\phi^{-1}(L)$ and $\gamma_{\left(\zeta_{2}, \zeta_{3}\right)}^{3}$, respectively. The above inequalities yield

$$
\omega\left(\beta_{\left(\zeta_{2}, \zeta_{3}\right)} \cap\left(R_{1} \cup R_{2}\right)^{c}\right) \leq c(d) \omega\left(\beta_{\left(\zeta_{0}, \zeta_{2}\right)} \cup \beta_{\left(\zeta_{1}, \zeta_{3}\right)}\right)
$$

Similarly, we can prove

$$
\omega\left(\beta_{\left(\zeta_{0}, \zeta_{2}\right)} \cap\left(R_{1} \cup R_{2}\right)^{c}\right) \leq c(d) \omega\left(\beta_{\left(\zeta_{0}, \zeta_{2}\right)} \cap\left(R_{1} \cup R_{2}\right)\right)
$$

and

$$
\omega\left(\beta_{\left(\zeta_{1}, \zeta_{3}\right)} \cap\left(R_{1} \cup R_{2}\right)^{c}\right) \leq c(d) \omega\left(\beta_{\left(\zeta_{1}, \zeta_{3}\right)} \cap\left(R_{1} \cup R_{2}\right)\right)
$$


Combining the above three inequalities, we obtain (4.1) for some constant $c(d)>0$ depending on $d$.

The next lemma relates 1-dimensional Hausdorff measure to harmonic measure on the boundary of a chord-arc domain. A chord-arc domain is a Jordan domain $\Omega$ such that there exists a constant $M>0$ satisfying

$$
\Lambda_{1}(\partial \Omega \cap B(x, r)) \leq M r
$$

for every $x \in \partial \Omega$ and $r>0$. Lemma 4.2 roughly means that the normalized linear measure of a set $E$ on a chord-arc boundary is small if harmonic measure of $E$ is small and vice versa. The detailed proof can be found in [JeKe 82] and [Pom 91].

Lemma 4.2. Let $\Omega$ be a chord-arc domain, and let $w_{0}$ be a point in $\Omega$ such that

$$
\operatorname{dist}\left(w_{0}, \partial \Omega\right) \approx \operatorname{diam}(\partial \Omega)
$$

Then there exist $C>0$ and $p>0$ depending on the chord-arc constant of $\Omega$ such that

$$
\frac{1}{C}\left(\frac{\Lambda_{1}(E)}{\Lambda_{1}(\partial \Omega)}\right)^{p} \leq \omega\left(w_{0}, E, \Omega\right) \leq C\left(\frac{\Lambda_{1}(E)}{\Lambda_{1}(\partial \Omega)}\right)^{1 / p}
$$

for all $E \subset \partial \Omega$.

Proof. Since $\Omega$ is a chord-arc domain and $\operatorname{dist}\left(w_{0}, \partial \Omega\right) \approx \operatorname{diam}(\partial \Omega), \omega$ and $\Lambda_{1}$ are $A^{\infty}$-equivalent on $\partial \Omega$ with $A^{\infty}$ constants depending on the chord-arc constant of $\Omega$. This implies that there exist $C>0$ and $p>0$ depending on the chord-arc constant of $\Omega$ such that

$$
\frac{1}{C}\left(\frac{\Lambda_{1}(E)}{\Lambda_{1}(\partial \Omega)}\right)^{p} \leq \omega\left(w_{0}, E, \Omega\right) \leq C\left(\frac{\Lambda_{1}(E)}{\Lambda_{1}(\partial \Omega)}\right)^{1 / p}
$$

for all $E \subset \partial \Omega$.

Using Lemma 4.2, we can prove

Lemma 4.3. Let $\Omega$ be a simply connected domain containing $[-1,1] \times[-1,0]$, and let $\Omega_{1}$ be a Lischitz domain of the form

$$
\Omega_{1}=\{(x, y):-1<x<1,-1<y<f(x)\}
$$

for some positive Lipschitz function $f(x)$ with Lipschitz norm bounded by $M$. Given any $\epsilon>0$, there exists $\delta(\epsilon, M)>0$ such that $\operatorname{dist}\left(\partial \Omega \cap \Omega_{1}, \partial \Omega_{1}\right)>\epsilon$ implies $\omega\left(\left(0,-\frac{1}{2}\right), \partial \Omega \cap \Omega_{1}, \Omega\right)>\delta$. 
Proof. Let $z$ be a point of $\partial \Omega \cap \Omega_{1}$ such that $\operatorname{dist}\left(z, \partial \Omega_{1}\right)=\epsilon$. For the ball $B(z, \epsilon)$ adjacent to the graph of $f$ (resp., the vertical lines of $\partial \Omega_{1}$ ), let $S$ be the half strip with width $\epsilon$ and with bottom on the horizontal (resp., vertical) diameter of $B\left(z, \frac{1}{2} \epsilon\right)$. (See Figure 7.) Then $\Omega_{1} \backslash S$ is a $C(M)$-Lipschitz domain and by Lemma 4.2 ,

$$
\omega\left(\left(0,-\frac{1}{2}\right), \partial \Omega \cap \Omega_{1}, \Omega\right) \geq C \omega\left(\left(0,-\frac{1}{2}\right), \text { bottom of } S, \Omega_{1} \backslash S\right)>\delta
$$

for some $\delta=\delta(\epsilon, M)>0$.

\section{Construction of a Lipschitz graph}

Sections 5 and 6 are the main parts of the proof of Theorem 2. In Section 5 , assuming $\Lambda_{1}(f(A))=0$ but $\omega(f(A))>0$, we construct a ball $B\left(f\left(\zeta_{0}\right), r_{0}\right)$ satisfying Lemma 3.1 and a Lipschitz graph $\Gamma$ in $B\left(f\left(\zeta_{0}\right), r_{0}\right)$ as in Proposition 5.1 below. Ultimately, the properties of $B\left(f\left(\zeta_{0}\right), r_{0}\right)$ and $\Gamma$ lead to a contradiction in Section 6. The arguments in Sections 5 and 6 depend mostly on elementary geometry and Lemmas 4.1, 4.2 and 4.3.

For $t(0<t<1)$ sufficiently close to 1 , take an interval $I \subset \partial \mathbb{D}$ and a ball $B\left(f\left(\zeta_{0}\right), r_{0}\right)$ satisfying properties (i)-(iv) of Lemma 3.1. Define

$$
A^{\prime}=\left\{\zeta \in I:|A \cap J| /|J| \geq t^{\prime} \text { for every interval } J \text { with } \zeta \in J \subset I\right\}
$$

for $t^{\prime}$ satisfying $0<t^{\prime}<t<1$. Then

$$
\begin{aligned}
I \backslash A^{\prime} & =\left\{\zeta \in I: \text { there exists } J \text { such that } \zeta \in J \subset I \text { and }|(I \backslash A) \cap J| /|J|>1-t^{\prime}\right\} \\
& =\left\{\zeta \in I: M\left(\chi_{(I \backslash A)}\right)(\zeta)>1-t^{\prime}\right\},
\end{aligned}
$$

where $M\left(\chi_{(I \backslash A)}\right)$ is the maximal function of $\chi_{(I \backslash A)}$. By the weak type 1-1 maximal function estimate and property (i) of Lemma 3.1, we obtain

$$
\left|I \backslash A^{\prime}\right| \leq \frac{C}{1-t^{\prime}}|I \backslash A| \leq \frac{C}{1-t^{\prime}}(1-t)|I|,
$$

for some $C>0$. With $t^{\prime}$ fixed and close to 1 , we can make $\left|I \backslash A^{\prime}\right| /|I|$ small by choosing $t$ sufficiently close to 1 .

Let $B(f(z), r)$ be a ball satisfying property (v) of Lemma 3.1. Then $\omega\left(f(z), \beta \backslash f\left(A^{\prime}\right), \Omega\right)$ is sufficiently small, i.e.,

$$
\omega\left(f(z), \beta \backslash f\left(A^{\prime}\right), \Omega\right) \ll 1,
$$

since

$$
\begin{aligned}
\omega\left(\beta \backslash f\left(A^{\prime}\right)\right) & \gtrsim \omega\left(w_{0}, \partial B\left(f(z), \frac{1}{2} r\right), \Omega \backslash B\left(f(z), \frac{1}{2} r\right)\right) \omega\left(f(z), \beta \backslash f\left(A^{\prime}\right), \Omega\right) \\
& \approx \omega(\beta) \omega\left(f(z), \beta \backslash f\left(A^{\prime}\right), \Omega\right)
\end{aligned}
$$


and

$$
\frac{\omega\left(\beta \backslash f\left(A^{\prime}\right)\right)}{\omega(\beta)}=\frac{\left|I \backslash A^{\prime}\right|}{|I|}
$$

was chosen to be sufficiently small. Formula (5.2) will be frequently used in Section 6.

To simplify the proof, we assume $r=\frac{1}{5} r_{0}$ and $f(z) \in \partial B\left(f\left(\zeta_{0}\right), \frac{2}{5} r_{0}\right)$ in (3.1). The proof in the general case is given at the end of Section 6 . The next proposition constructs a Lipschitz graph $\Gamma$ in $B\left(f\left(\zeta_{0}\right), r_{0}\right)$.

Proposition 5.1. With $A$ and $A^{\prime}$ as before and $\Lambda_{1}(f(A))=0$ but $\omega(f(A))>0$, let $f(z) \in \partial B\left(f\left(\zeta_{0}\right), \frac{2}{5} r_{0}\right)$ and let

$$
B\left(f(z), \frac{1}{5} r_{0}\right) \subset \Omega \cap B\left(f\left(\zeta_{0}\right), r_{0}\right) .
$$

Then there exist a line segment $L \subset \Omega \cap B\left(f\left(\zeta_{0}\right), r_{0}\right)$ and a Lipschitz graph $\Gamma$ over $L$ such that

(i) $|L| \geq \frac{1}{6} r_{0}$;

(ii) assuming $L \subset \mathbb{R}$ a Lipschitz graph $\Gamma$ consists of horizontal segments $I_{i}$ and line segments $l_{j}$ with slope of \pm 98000 which connect the endpoints of $I_{i}$ and make the area below the graph as big as possible;

(iii) $\sum\left|I_{i}\right| \ll|L|$;

(iv) $I_{i} \cap f\left(A^{\prime}\right) \neq \emptyset$;

(v) the region between $L$ and $\Gamma$ is contained in $B\left(f\left(\zeta_{0}\right), r_{0}\right)$ and does not intersect $f\left(A^{\prime}\right)$.

Proof. First, we describe the basic construction of $\Gamma$ when $L \subset \mathbb{R}$ is given. Assume $f\left(A^{\prime}\right)$ is compact. Since $\Lambda_{1}\left(f\left(A^{\prime}\right)\right)=0$, there exist finite number of balls $\left\{D_{i}\right\}$ such that

$$
\sum_{i} \operatorname{rad}\left(D_{i}\right) \ll|L| \text { and } f\left(A^{\prime}\right) \subset \bigcup_{i} D_{i}
$$

Let $p$ be the projection onto $L$; then there exist disjoint intervals $\left\{\left(p_{j}, q_{j}\right)\right\}$ on $L$ such that

$$
\bigcup_{i}\left(p\left(D_{i}\right)\right)=\bigcup_{j}\left(p_{j}, q_{j}\right)
$$

since $\bigcup_{i}\left(p\left(D_{i}\right)\right)$ is open.

Let $x_{1}$ be one of the lowest points in

$$
f\left(A^{\prime}\right) \cap p^{-1}(L)
$$


and let $\left(p_{1}, q_{1}\right)$ denote the interval containing $p\left(x_{1}\right)$. We construct the horizontal line segment $I_{1}$ such that $x_{1} \in I_{1}$ and

$$
p\left(I_{1}\right)=\left(p_{1}, q_{1}\right) .
$$

Next, let $x_{2}$ be one of the lowest points in

$$
\left\{x \in f\left(A^{\prime}\right) \cap p^{-1}\left(L \backslash\left(p_{1}, q_{1}\right)\right): \mid \text { slope of any line connecting } x \text { and } I_{1} \mid \leq 98000\right\} \text {. }
$$

Let $\left(p_{2}, q_{2}\right)$ denote the interval containing $p\left(x_{2}\right)$ and construct the maximal, horizontal line segment $I_{2}$ such that

$$
x_{2} \in I_{2}, \quad p\left(I_{2}\right) \subset\left(p_{2}, q_{2}\right)
$$

and

$\mid$ slope of any line connecting $I_{1}$ and $I_{2} \mid \leq 98000$.

After inductively constructing $\left\{I_{i}\right\}$, we choose line segments $l_{j}$ with slope of \pm 98000 which connect the endpoints of $\left\{I_{i}\right\}$ and make the area below the graph of $\left\{I_{i}\right\} \cup\left\{l_{j}\right\}$ as big as possible. Call this Lipschitz graph $\Gamma$; then clearly

$$
I_{i} \cap f\left(A^{\prime}\right) \neq \emptyset,
$$

and the region between $L$ and $\Gamma$ does not intersect $f\left(A^{\prime}\right)$.

Now we construct a line segment $L$ such that $|L| \geq \frac{1}{6} r_{0}$ and for $\Gamma$ given by the basic construction,

$$
\Gamma \subset B\left(f\left(\zeta_{0}\right), r_{0}\right) \text {. }
$$

For the line $V$ passing through $f(z)$ and $f\left(\zeta_{0}\right)$, let

$$
S=\left\{x: \operatorname{dist}(x, V) \leq \frac{1}{6} r_{0}\right\}
$$

and let $L_{S}, R_{S}$ be the left- and right-half plane adjacent to $S$, respectively. We divide into three cases as follows.

Case 1. There exists a line segment $L$ contained in the diameter of $B\left(f(z), \frac{1}{5} r_{0}\right)$ perpendicular to $V$ such that

$$
|L| \geq \frac{1}{6} r_{0}
$$

and for $\Gamma$ given by the basic construction,

$$
\Gamma \subset B\left(f\left(\zeta_{0}\right), r_{0}\right)
$$




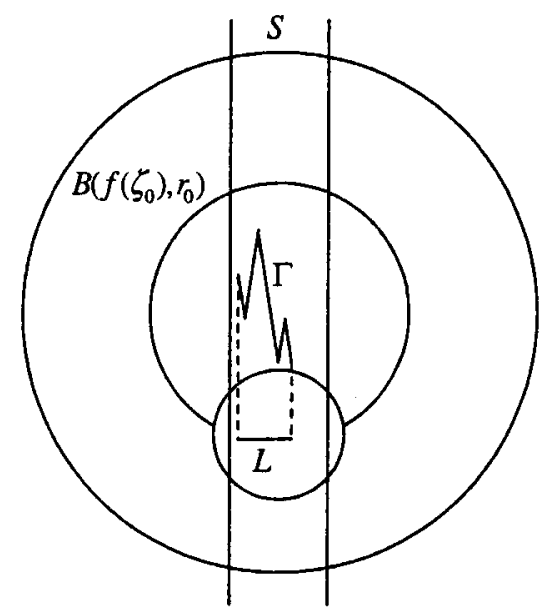

Figure 3. $L$ and $\Gamma$ of Case 1.

Case 2. There does not exist $L$ of Case 1 and

$$
\partial \Omega \cap B\left(f\left(\zeta_{0}\right), \frac{2}{5} r_{0}\right) \cap W_{S}=\emptyset
$$

where $W_{S}=L_{S}$ or $W_{S}=R_{S}$.

Case 3. There does not exist $L$ of Case 1,

$$
\partial \Omega \cap B\left(f\left(\zeta_{0}\right), \frac{2}{5} r_{0}\right) \cap L_{S} \neq \emptyset \quad \text { and } \quad \partial \Omega \cap B\left(f\left(\zeta_{0}\right), \frac{2}{5} r_{0}\right) \cap R_{S} \neq \emptyset .
$$

Case 1 is the simplest, since $L$ and $\Gamma$ of Case 1 satisfy the desired properties of Proposition 5.1. (See Figure 3.)

In Case 2, let $L_{1}$ be the diameter of $B\left(f(z), \frac{1}{5} r_{0}\right)$ perpendicular to $V$ and let $\Gamma_{1}$ be the Lipschitz graph given by the basic construction with $L=L_{1}$. Define

$$
\Omega^{\prime}=\left(S \cap\left\{x: x \text { is between } L_{1} \text { and } \Gamma_{1}\right\}\right) \cup B\left(f(z), \frac{1}{5} r_{0}\right) .
$$

(See Figure 4.) Lemma 4.3 implies that for some fixed small constant $c>0$,

$$
\partial \Omega \cap\left\{x \in \Omega^{\prime}: \operatorname{dist}\left(x, \partial \Omega^{\prime}\right) \geq c r_{0}\right\}=\emptyset,
$$

since

$$
f\left(A^{\prime}\right) \cap \Omega^{\prime}=\emptyset \quad \text { and } \quad \omega\left(f(z), \beta \backslash f\left(A^{\prime}\right), \Omega\right) \ll 1 .
$$

In Case 2, $\Gamma_{1}$ leaves $B\left(f\left(\zeta_{0}\right), r_{0}\right)$ on both the left and right halves of $L_{1}$. Thus $\Gamma_{1}$ has two spikes, one on either side of $f\left(\zeta_{0}\right)$, which $\beta$ cannot cross; hence $\beta$ 

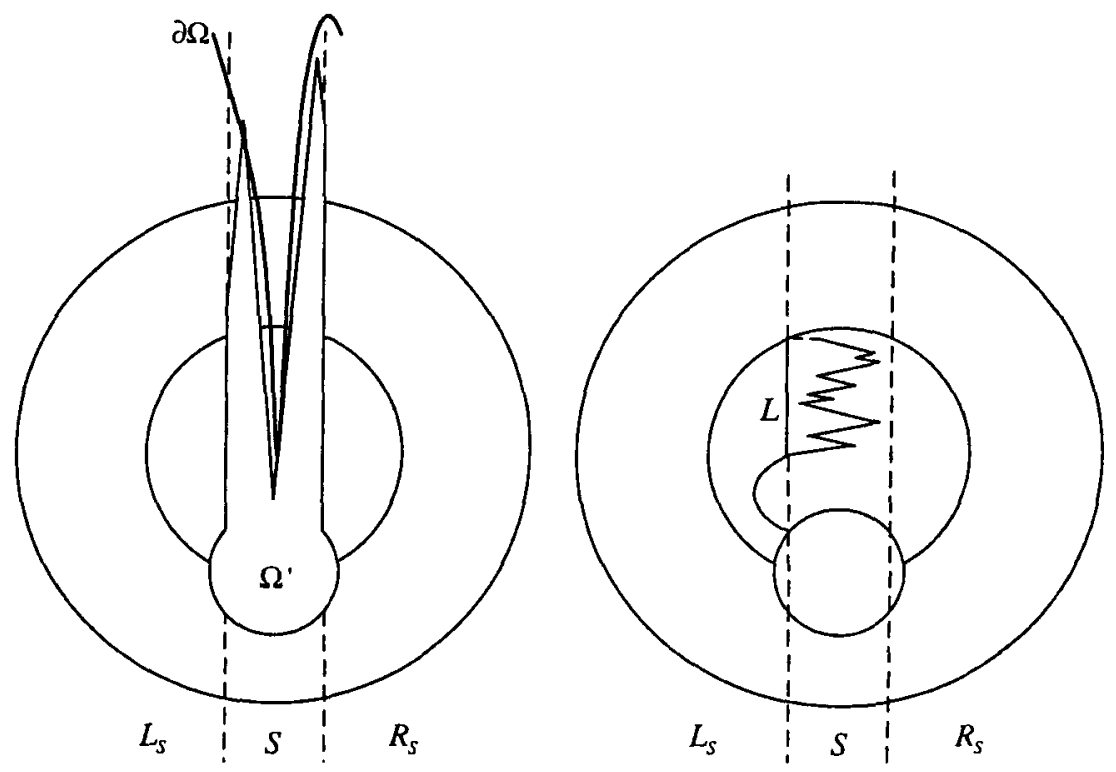

Figure 4. $L$ and $\Gamma$ of Case 2, when $W_{S}=L_{S}$.

must leave $B\left(f\left(\zeta_{0}\right), r_{0}\right)$ through the strip $S$. Now, we let $L$ be the upper half line segment of $\partial W_{S} \cap B\left(f\left(\zeta_{0}\right), \frac{2}{5} r_{0}\right)$ and let $\Gamma$ be the Lipschitz graph given by the basic construction over $L$. Let $\Omega^{\prime \prime}$ be the Lipschitz domain containing $B\left(f(z), \frac{1}{5} r_{0}\right)$ with $\Gamma \subset \partial \Omega^{\prime \prime}$. (See Figure 4.) Since (5.4) holds with $\Omega^{\prime}$ replaced by $\Omega^{\prime \prime}$ and $\beta$ leaves the ball through the strip $S$, the Lipschitz graph $\Gamma$ constructed over $L$ must be contained in $B\left(f\left(\zeta_{0}\right), r_{0}\right)$.

Finally, we consider Case 3 and show that, in fact, it cannot occur. In Case 3, there exist

$$
q_{1} \in \beta \cap B\left(f\left(\zeta_{0}\right), \frac{2}{5} r_{0}\right) \cap L_{S} \quad \text { and } \quad q_{2} \in \beta \cap B\left(f\left(\zeta_{0}\right), \frac{2}{5} r_{0}\right) \cap R_{S},
$$

by property (ii) of Lemma 3.1. Also, by the same argument as in Case 2,

$$
\partial \Omega \cap\left\{x \in \Omega^{\prime}: \operatorname{dist}\left(x, \partial \Omega^{\prime}\right) \geq c r_{0}\right\}=\emptyset
$$

for $\Omega^{\prime}$ defined in Case 2. (See Figure 5.) By choosing the constant $c>0$ of the above equality sufficiently small, we can take ring domains $T_{k, 1}, T_{k, 2}(k=1,2, \ldots)$ as in Figure 5, which satisfy

(i) $T_{k, i}$ is contained in $S \cap B\left(f\left(\zeta_{0}\right), \frac{99}{100} r_{0}\right)$;

(ii) $T_{k, 1}$ and $T_{k, 2}$ are bounded by rectangles and neighboring as in Lemma 4.1; 
(iii) $f\left(\zeta_{0}\right)$ is contained in the bounded component of $\left(T_{k, 1} \cup T_{k, 2}\right)^{c}$;

(iv) every region between the vertical sides or bottoms of the inner and outer rectangles of $\partial T_{k, i}$ is contained in $\Omega$;

(v) $\bmod \left(T_{k, i}\right)$ is bounded below by a small constant $d>0$ depending on the Lipschitz constant of $\Gamma$.

We also take annuli $U_{k, 1}, U_{k, 2}(k=1,2, \ldots)$ as in Figure 5 , which satisfy

(i) $U_{k, 1}$ and $U_{k, 2}$ are neighboring annuli in $B\left(f\left(\zeta_{0}\right), \frac{99}{100} r_{0}\right) \backslash B\left(f\left(\zeta_{0}\right), \frac{2}{5} r_{0}\right)$, with centers at $f\left(\zeta_{0}\right)$;

(ii) $\bmod \left(U_{k, i}\right)=d$.

Let $c(d)$ be the constant satisfying (4.1) of Lemma 4.1 so that

$$
\frac{\omega\left(\alpha \cap\left(R_{1} \cup R_{2}\right)\right)}{\omega(\alpha)} \geq c(d)>0
$$

for $R_{i}=T_{k, i}$ or $R_{i}=U_{k, i}$ and $\alpha \subset \partial \Omega$ satisfying the hypothesis of Lemma 4.1. Choose $t>0$ greater than $1-c(d)$. Let $J$ be the subarc of $\partial \mathbb{D}$ such that $J$ contains $\zeta_{0}$ and $f(J)$ has a circular crosscut on $\Omega \cap$ (outer boundary of $R_{1} \cup R_{2}$ ). Then (i) of Lemma 3.1 implies

$$
\begin{aligned}
t \leq \frac{|J \cap A|}{|J|} & =\frac{\omega(f(J) \cap f(A))}{\omega(f(J))} \\
& \leq \frac{\omega\left(f(A) \cap\left(R_{1} \cup R_{2}\right)\right)}{\omega(f(J))}+\frac{\omega\left(f(J) \cap\left(R_{1} \cup R_{2}\right)^{c}\right)}{\omega(f(J))} \\
& \leq \frac{\omega\left(f(A) \cap\left(R_{1} \cup R_{2}\right)\right)}{\omega(f(J))}+1-c(d) .
\end{aligned}
$$

Since $t$ is greater than $1-c(d)$,

$$
f(A) \cap\left(R_{1} \cup R_{2}\right) \neq \emptyset
$$

Using property (iv) of $T_{k, i}$ and (iv) of Lemma 3,1, we can construct disjoint balls $B_{j}$ with centers in $f(A)$ such that

$$
\sum \operatorname{rad}\left(B_{j}\right) \geq \frac{1}{2} \cdot\left(2 \cdot\left(\frac{3}{5}-\frac{1}{100}\right)+\left(1-\frac{1}{100}\right)\right) r_{0}>(1.01) r_{0}
$$

This contradicts (iii) of Lemma 3.1. Hence Case 3 cannot occur. 


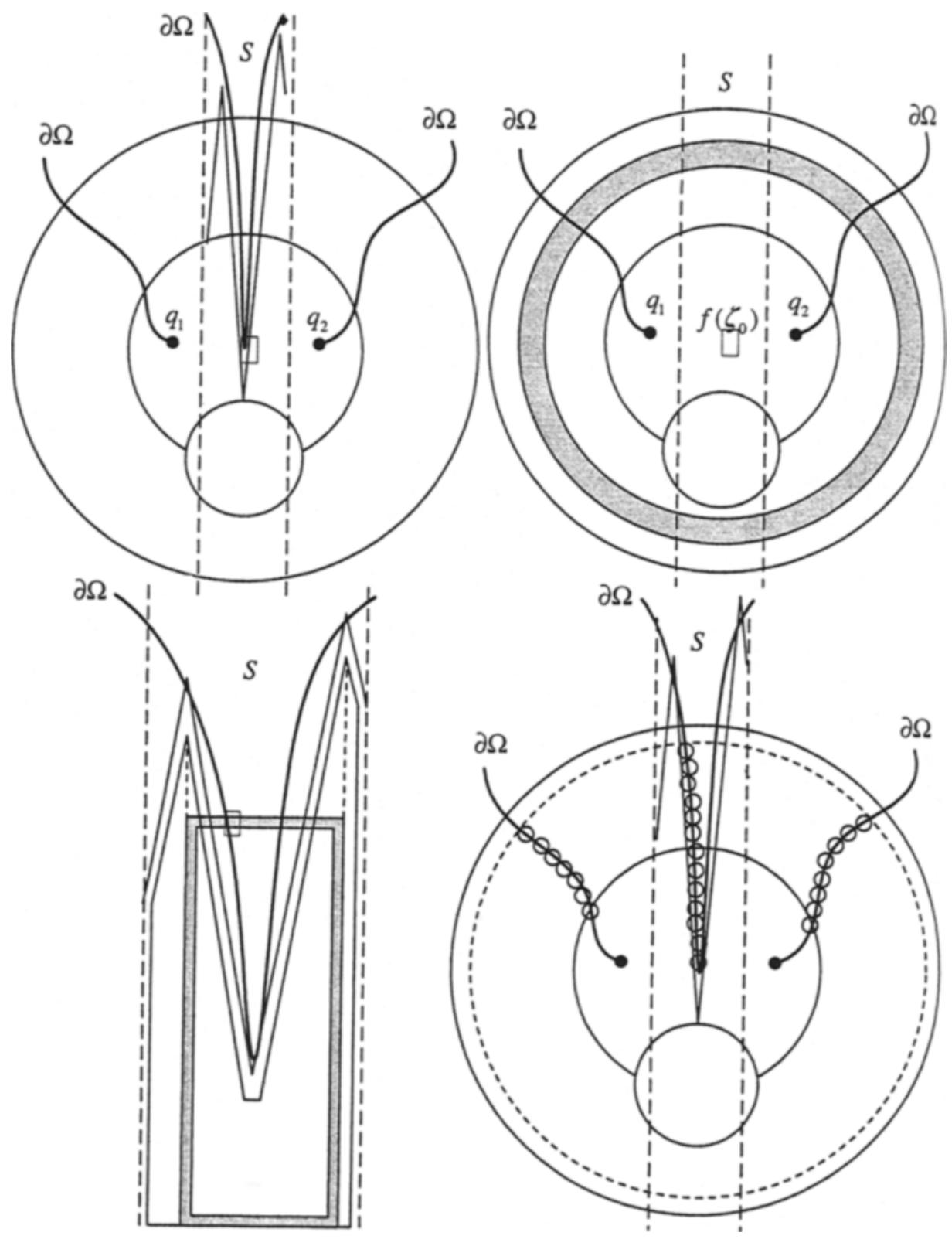

Figure 5. Case 3 and ring domains. 


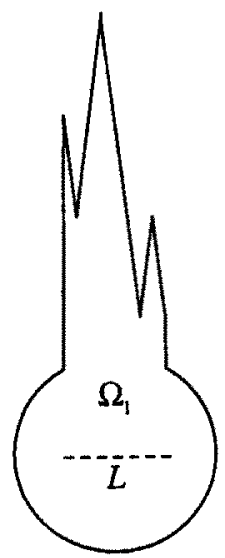

Case 1

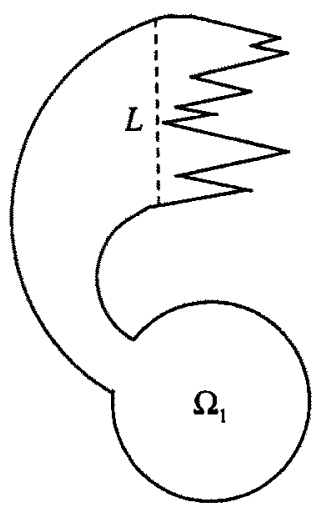

Case 2

Figure 6. $\Omega_{1}$ and $L$ in Case 1 and Case 2 of Section 5.

\section{Contradicting a geometric property of $f(A)$}

In this final section, we construct a disjoint collection of balls $B_{k}$ in $B\left(f\left(\zeta_{0}\right), r_{0}\right)$ such that

$$
\operatorname{center}\left(B_{k}\right) \in f(A) \text { and } \sum \operatorname{rad}\left(B_{k}\right)>(1.01) r_{0} .
$$

This contradicts property (iii) of Lemma 3.1 and hence completes the proof of Theorem 2.

For the construction of $\left\{B_{k}\right\}$, we need an observation about the behavior of $\partial \Omega$ near $\Gamma$. Let $\Omega_{1}$ be a union of $B\left(f(z), \frac{1}{5} r_{0}\right)$, the region between $L$ and $\Gamma$, and a subregion of $\Omega \cap B\left(f(z), \frac{2}{5} r_{0}\right)$ such that $\Omega_{1}$ is a chord-arc domain with chord-arc constant depending only on the Lipschitz constant of $\Gamma$. (See Figure 6.) Also, let $\Omega_{2}$ be the component of $\Omega \cap \Omega_{1}$ containing $B\left(f(z), \frac{1}{5} r_{0}\right)$. Since $f\left(A^{\prime}\right) \cap \Omega_{1}=\emptyset$,

$$
\omega\left(f(z), \partial \Omega_{2} \cap \Omega_{1}, \Omega_{2}\right) \leq \omega\left(f(z), \beta \backslash f\left(A^{\prime}\right), \Omega\right) \ll 1,
$$

where the right inequality follows from (5.2). For $y \in \partial \Omega_{2} \cap \Omega_{1}$, let $B_{y}$ be the ball adjacent to $\partial \Omega_{1}$ with center at $y$. Assume that for some $c>0$,

$$
\left|\bigcup_{y \in \partial \Omega_{2} \cap \Omega_{1}} p\left(B_{y}\right)\right|>c|L|
$$

where $p$ is the projection onto $L$. Then by Besicovitch's covering lemma, there exist disjoint intervals $\left\{p\left(B_{y_{i}}\right)\right\}$ satisfying

$$
\left|\bigcup_{i} p\left(B_{y_{i}}\right)\right|>\frac{1}{2} c|L|
$$




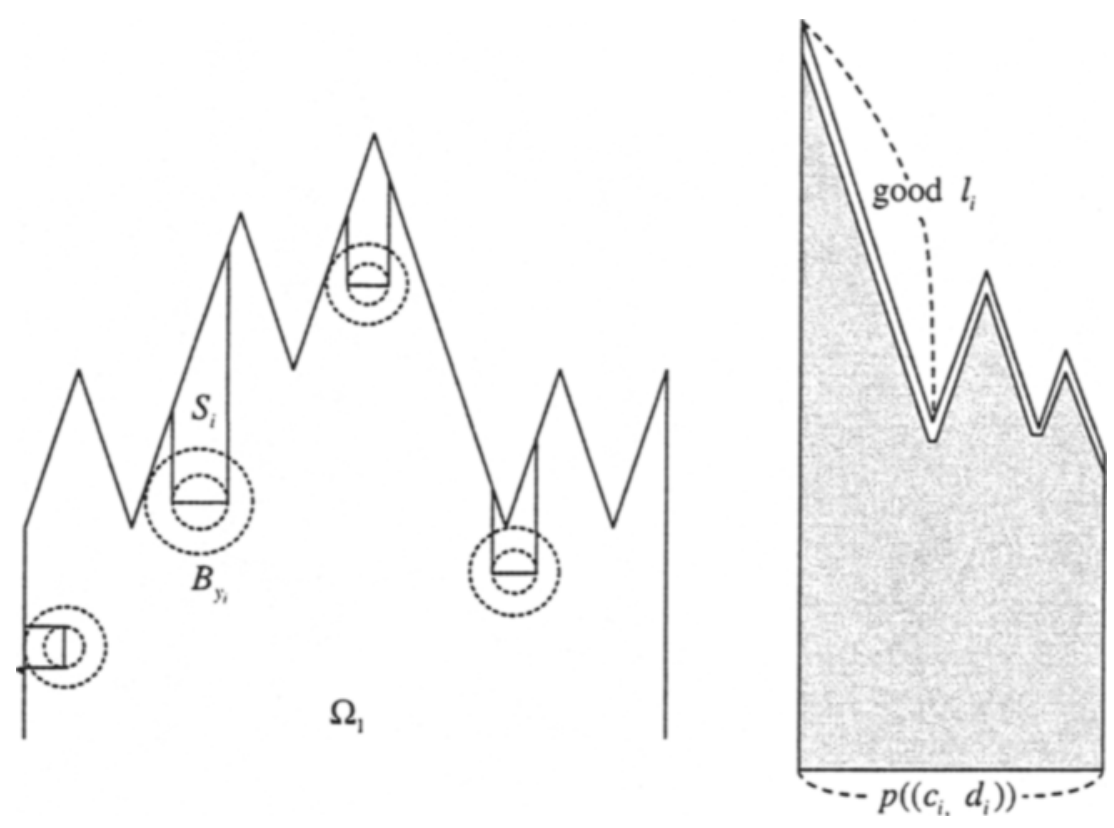

Figure 7. $S_{i}$ and good $l_{i}$.

For $B_{y_{i}}$ adjacent to $\Gamma$, let $S_{i}$ be the half strip going up from the horizontal diameter of $\frac{1}{2} B_{y_{i}}$. (See Figure 7.) Also for $B_{y_{i}}$ adjacent to the vertical edges of $\partial \Omega_{1}$, let $S_{i}$ be the half strip from the vertical diameter of $\frac{1}{2} B_{y_{i}}$ to the adjacent vertical edge. Then

$$
\Omega_{1} \backslash \bigcup_{i}\left(S_{i} \cup \frac{1}{2} B_{y_{i}}\right)
$$

is a chord-arc domain with chord-arc constant depending on the Lipschitz constant of $\Gamma$. Hence by (6.2) and Lemma 4.2,

$\omega\left(f(z), \bigcup_{i} \partial\left(\frac{1}{2} B_{y_{i}}\right), \Omega_{1} \backslash \bigcup_{i} \frac{1}{2} B_{y_{i}}\right) \geq \omega\left(f(z), \bigcup_{i} \partial\left(\frac{1}{2} B_{y_{i}}\right), \Omega_{1} \backslash \bigcup_{i}\left(S_{i} \cup \frac{1}{2} B_{y_{i}}\right)\right)>c^{\prime}$

for some $c^{\prime}>0$. Also by Beurling's projection theorem,

$$
\omega\left(w, \partial \Omega_{2}, B_{y_{i}} \backslash \partial \Omega_{2}\right) \geq C
$$

for every $w \in \partial\left(\frac{1}{2} B_{y_{i}}\right)$. Now (6.3) and (6.4) show that

$$
\omega\left(f(z), \partial \Omega_{2} \cap \Omega_{1}, \Omega_{2}\right) \geq c^{\prime \prime}
$$

for some $c^{\prime \prime}>0$. This contradicts $(6.1)$; hence

$$
\left|\bigcup_{y \in \partial \Omega_{2} \cap \Omega_{1}} p\left(B_{y}\right)\right| \ll|L| .
$$




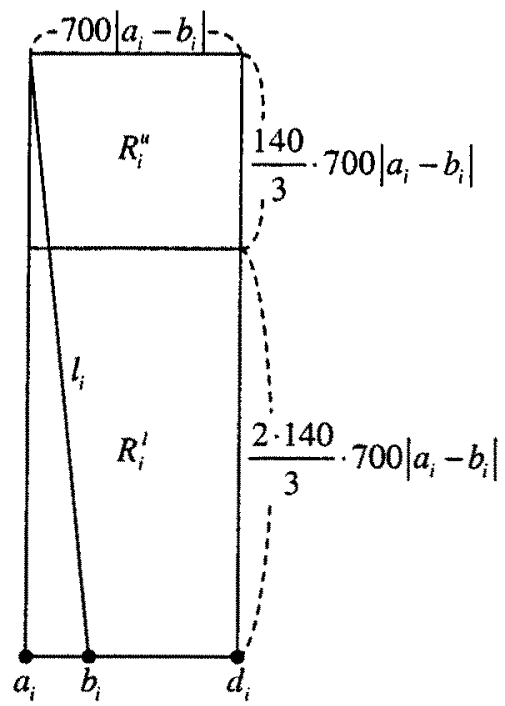

Figure $8 . R_{i}, R_{i}^{u}$ and $R_{i}^{l}$.

Next, we make several definitions useful for the construction of $\left\{B_{k}\right\}$. For each $l_{i} \subset \Gamma$ with slope \pm 98000 , let $\left(a_{i}, b_{i}\right)$ be the interval parallel to $L$ such that $\left\{a_{i}, b_{i}\right\}$ intersects the lower endpoint of $l_{i}$ and $p\left(\left(a_{i}, b_{i}\right)\right)=p\left(l_{i}\right)$. (See Figure 8.) When the slope of $l_{i}$ is -98000 , let $c_{i}=a_{i}$ and $d_{i}=a_{i}+700\left|a_{i}-b_{i}\right|$ and let $R_{i}$ be the rectangle with bottom $\left(c_{i}, d_{i}\right)$ and height $140\left|c_{i}-d_{i}\right|$. When the slope of $l_{i}$ is 98000 , let $c_{i}=b_{i}-700\left|a_{i}-b_{i}\right|$ and $d_{i}=b_{i}$ and similarly define the rectangle $R_{i}$. Also let $R_{i}^{u}$ denote the upper subrectangle of $R_{i}$ with height $\frac{1}{3} \cdot 140 \cdot 700\left|a_{i}-b_{i}\right|$ and let $R_{i}^{l}$ denote the lower subrectangle of $R_{i}$ with height $\frac{2}{3} \cdot 140 \cdot 700\left|a_{i}-b_{i}\right|$.

Fix a small constant $c>0$. We say that $l_{i}$ is good if

$$
\left|p\left(\left(c_{i}, d_{i}\right)\right) \cap\left(\bigcup_{\substack{y \in \theta \Omega_{2} \\ \cap \Omega_{1}}} p\left(B_{y}\right)\right)\right|<c\left|a_{i}-b_{i}\right| .
$$

Otherwise, say that $l_{i}$ is bad. If $l_{i}$ is good,

(6.7) $\left\{x: x\right.$ is between $\Gamma$ and $L, p(x) \in p\left(\left(c_{i}, d_{i}\right)\right)$, $\left.\operatorname{dist}(x, \Gamma) \geq c\left|a_{i}-b_{i}\right|\right\} \subset \Omega$.

(The shaded region of Figure 7 is contained in $\Omega$.) Also by (6.5),

$$
\sum\left\{\left|a_{i}-b_{i}\right| \mid l_{i} \text { is bad }\right\} \ll|L| .
$$

We consider three cases according to the geometry of $\Gamma$ and $f\left(A^{\prime}\right)$ and construct $\left\{B_{k}\right\}$ for each case. Assume $|L|=1$. 


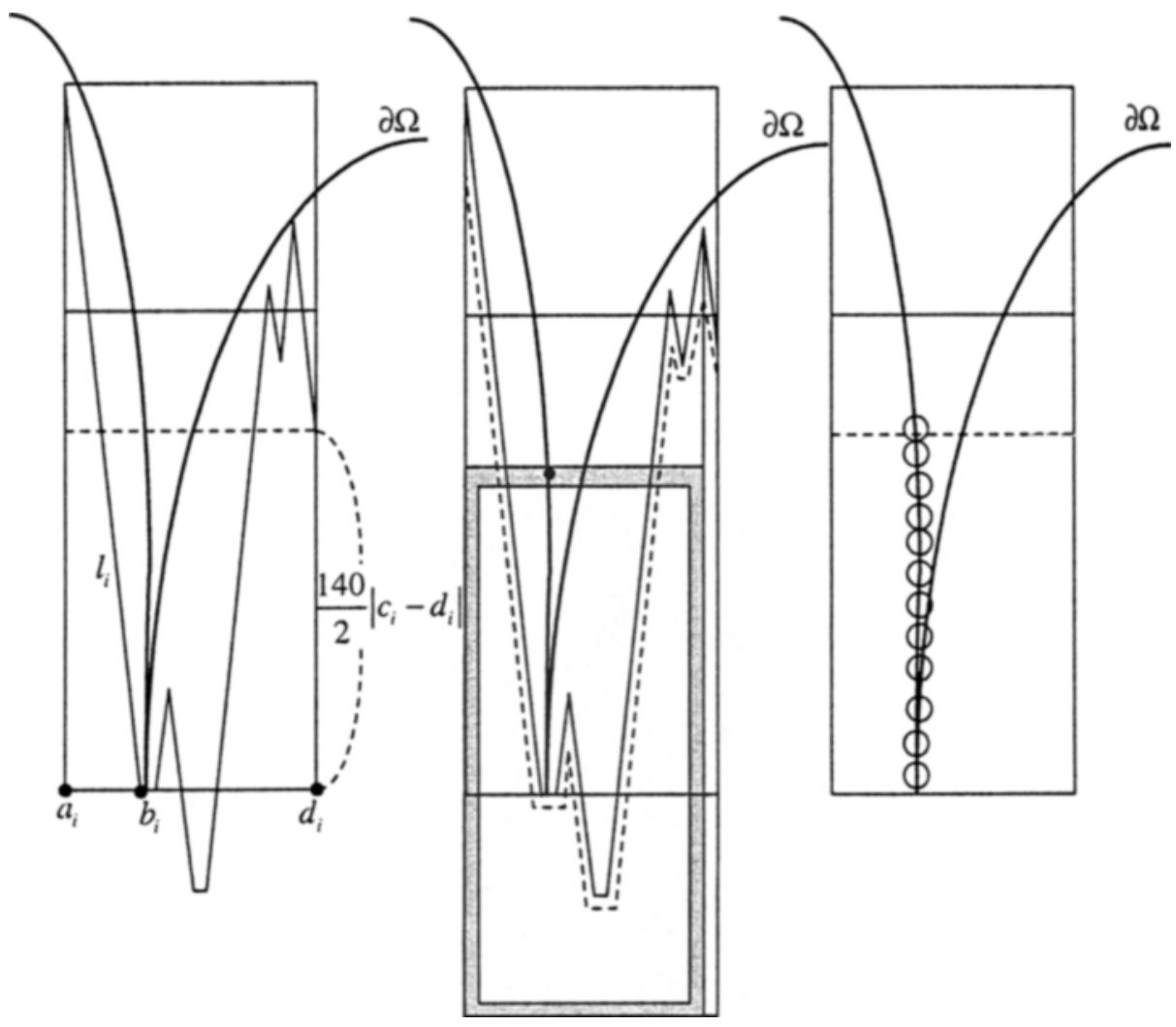

Figure 9. Case I and ring domains.

Case I. $\left|\bigcup\left\{p\left(\left(c_{i}, d_{i}\right)\right): R_{i}^{u} \cap\left(\partial \Omega_{1} \backslash l_{i}\right) \neq \emptyset\right\}\right| \geq \frac{12}{30}$.

In Case I, there exist disjoint intervals $\left\{p\left(\left(c_{i_{j}}, d_{i_{j}}\right)\right)\right\}$ such that

$$
\sum\left|c_{i_{j}}-d_{i_{j}}\right| \geq \frac{12}{60}
$$

and by (6.8),

$$
\sum\left\{\mid c_{i_{j}}-d_{i_{j}} \| l_{i_{j}} \text { is good }\right\} \geq \frac{11}{60} .
$$

Let $I_{i_{j}}$ denote the horizontal segment of $\Gamma$ adjacent to $\left(a_{i_{j}}, b_{i_{j}}\right)$. Then by (5.3) and (6.7), the arc $\beta$ comes down from the top of $R_{i_{j}}^{l}$ to a point in $I_{i_{j}} \cap f\left(A^{\prime}\right)$ and then passes through the top of $R_{i_{j}}^{l}$. (See Figure 9.) We take nested ring domains $T_{k, 1}$, $T_{k, 2}(k=1,2, \ldots)$ such that:

(i) $T_{k, i}$ is located under the upper half subrectangle of $R_{i_{j}}$;

(ii) $T_{k, 1}$ and $T_{k, 2}$ are bounded by rectangles and neighboring as in Lemma 4.1; 
(iii) $I_{i_{j}}$ is contained in the bounded component of $\left(T_{k, 1} \cup T_{k, 2}\right)^{c}$;

(iv) every region between the vertical sides or bottoms of the inner and outer rectangles of $\partial T_{k, i}$ is contained in $\Omega$;

(v) $\bmod \left(T_{k, i}\right)$ is bounded below by a small constant $d>0$ depending on the Lipschitz constant of $\Gamma$. (See Figure 9.)

Choose $t^{\prime}>0$ greater than $1-c(d)$ for the constant $c(d)$ satisfying (4.1). Then by Lemma 4.1, (5.1) and (6.7), we can construct disjoint balls $B_{k}^{j}$ in $R_{i_{j}}^{l}$ with centers in $f(A)$ such that

$$
\sum_{k} \operatorname{rad}\left(B_{k}^{j}\right) \geq 35\left|c_{i_{j}}-d_{i_{j}}\right| .
$$

Observe that $\left\{B_{k}^{j}\right\}_{j, k}$ is disjoint and

$$
\sum_{j, k} \operatorname{rad}\left(B_{k}^{j}\right) \geq 35 \cdot \frac{11}{60}>(1.01) \cdot 6=(1.01) \cdot 6 \cdot|L| \geq(1.01) r_{0} .
$$

This gives the desired contradiction to property (iii) of Lemma 3.1.

Case II. $\left|\cup\left\{\left(a_{i}, b_{i}\right): R_{i}^{u} \cap\left(\partial \Omega_{1} \backslash l_{i}\right)=\emptyset, R_{i}^{m} \cap f\left(A^{\prime}\right) \neq \emptyset\right\}\right| \geq \frac{2}{30}$, where $R_{i}^{m}$ is the contiguous subrectangle of $R_{i}^{u}$ with $\operatorname{width}\left(R_{i}^{m}\right)=\frac{2}{3} \operatorname{width}\left(R_{i}^{u}\right)$ and height $\left(R_{i}^{m}\right)=\frac{1}{3}$ height $\left(R_{i}^{u}\right)$. (See Figure 10.)

By (6.8), we get

$$
\mid \bigcup\left\{\left(a_{i}, b_{i}\right) \text { of Case II }: l_{i} \text { is } \operatorname{good}\right\} \mid \geq \frac{2}{31}
$$

and

$$
\| \bigcup\left\{\left(a_{i}, b_{i}\right) \text { of Case II : } l_{i} \text { is good and slope }\left(l_{i}\right)=w\right\} \mid \geq \frac{1}{31} \text {, }
$$

where $w=98000$ or $w=-98000$. If $\left(a_{i}, b_{i}\right)$ and $\left(a_{j}, b_{j}\right)(i \neq j)$ satisfy the conditions in $(6.9)$, then

$$
R_{i}^{u} \cap R_{j}^{u}=\emptyset
$$

For $\left(a_{i}, b_{i}\right)$ of $(6.9)$, we construct disjoint balls $B_{k}^{i}$ in $R_{i}^{u}$ as follows.

If there exists $q \in R_{i}^{m} \cap f\left(A^{\prime}\right)$ in the half subrectangle of $R_{i}^{m}$ far from $l_{i}$, then let

$$
B_{1}^{i}=B\left(q, \frac{1}{2} \text { width }\left(R_{i}^{m}\right)\right)
$$

Then

$$
B_{1}^{i} \subset R_{i}^{u}
$$



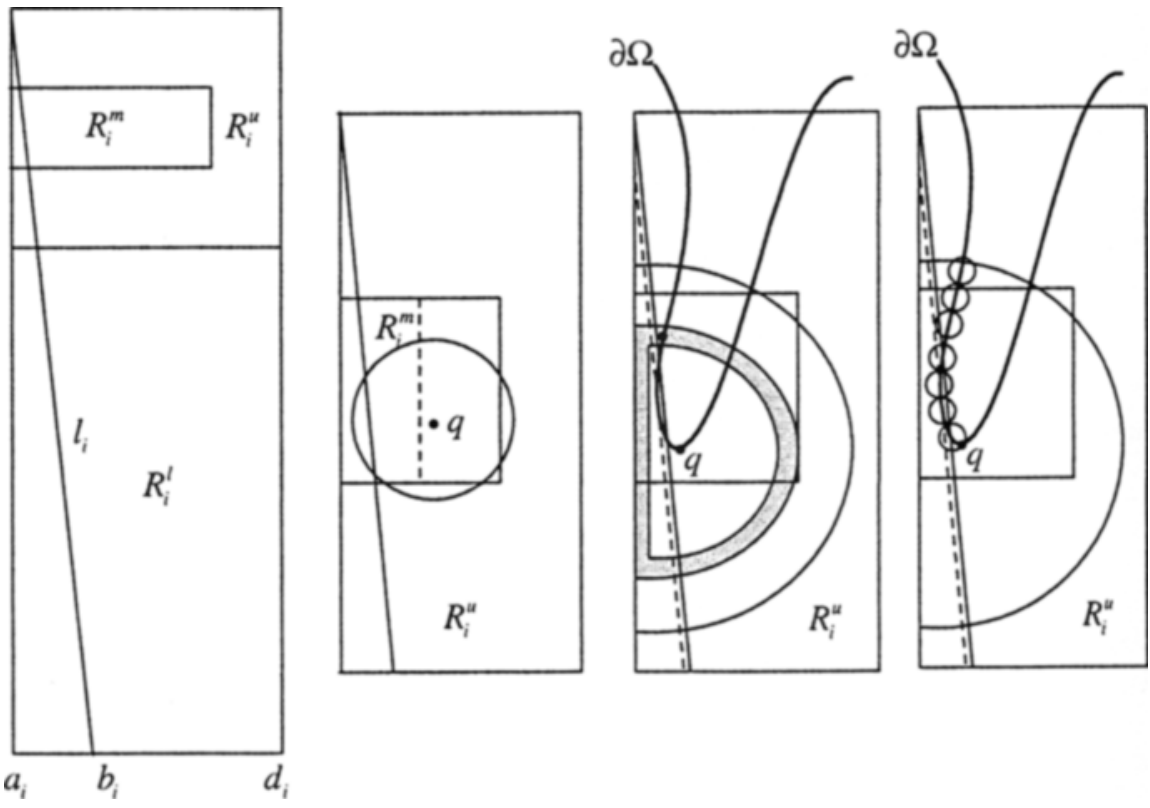

Figure 10. Case II and ring domains.

and

$$
\frac{1}{2} \operatorname{width}\left(R_{i}^{m}\right)=\frac{1}{3} \cdot 700 \cdot\left|a_{i}-b_{i}\right| .
$$

Otherwise, take $q^{\prime} \in R_{i}^{m} \cap f\left(A^{\prime}\right)$ in the other half subrectangle of $R_{i}^{m}$ and take ring domains $T_{k, 1}, T_{k, 2}(k=1,2, \ldots)$ such that

(i) $T_{k, 1}$ and $T_{k, 2}$ are contained in $B\left(q^{\prime}\right.$, width $\left.\left(R_{i}^{m}\right)\right) \cap R_{i}^{u}$ and neighboring as in Lemma 4.1;

(ii) $T_{k, j}$ is either an annulus centered at $q^{\prime}$ or obtained by replacing some arcs of the annulus by vertical line segments;

(iii) the region between the vertical line segments of $\partial T_{k, j}$ is contained in $\Omega$;

(iv) $\bmod \left(T_{k, j}\right)$ is bounded below by a small constant $d>0$ depending on the Lipschitz constant of $\Gamma$. (See Figure 10.)

Then by the same reasoning as in Case I, we can construct disjoint balls $B_{k}^{i} \subset R_{i}^{u}$ with centers in $f(A)$ such that

$$
\sum_{k} \operatorname{rad}\left(B_{k}^{i}\right) \geq \frac{1}{2} \operatorname{width}\left(R_{i}^{m}\right)=\frac{1}{3} \cdot 700 \cdot\left|a_{i}-b_{i}\right|
$$


By (6.10), $\left\{B_{k}^{i}\right\}_{i, k}$ is disjoint and

$$
\sum_{i, k} \operatorname{rad}\left(B_{k}^{i}\right) \geq \frac{1}{3} \cdot 700 \cdot \frac{1}{31}>(1.01) \cdot 6 \geq(1.01) r_{0} .
$$

Case III. $\left|\cup\left\{\left(a_{i}, b_{i}\right): R_{i}^{u} \cap\left(\partial \Omega_{1} \backslash l_{i}\right)=\emptyset, R_{i}^{m} \cap f\left(A^{\prime}\right)=\emptyset\right\}\right| \geq \frac{15}{30}$.

For $\left(a_{i}, b_{i}\right)$ of Case III, let $\frac{1}{2} R_{i}^{m}$ be the contiguous subrectangle of $R_{i}^{m}$ with width and height a half the width and height of $R_{i}^{m}$. (See Figure 11.) First, we show that

$$
\text { |U }\left\{\left(a_{i}, b_{i}\right) \text { of Case III : } \frac{1}{2} R_{i}^{m} \cap \partial \Omega \neq \emptyset\right\} \mid \ll 1 .
$$

For the proof of $(6.11)$, let

$$
\left\{\left(a_{j}, b_{j}\right): j \in J\right\}=\left\{\left(a_{i}, b_{i}\right) \text { of Case III : } \frac{1}{2} R_{i}^{m} \cap \partial \Omega \neq \emptyset\right\} .
$$

Since $\Omega_{1} \cap f\left(A^{\prime}\right)=R_{i}^{m} \cap f\left(A^{\prime}\right)=\emptyset$, we obtain for some $C>0$,

$$
\begin{aligned}
& \omega\left(f(z), \beta \backslash f\left(A^{\prime}\right), \Omega\right) \\
& \geq \omega\left(f(z), \bigcup_{j \in J}\left(l_{j} \cap \frac{1}{2} R_{j}^{m}\right), \Omega_{1}\right) \cdot \min _{\substack{y \in l_{j} \cap \frac{1}{2} R_{j}^{m} \\
j \in j}} \omega\left(y, \partial \Omega \cap R_{j}^{m},\left(\Omega_{1} \cup R_{j}^{m}\right) \backslash \partial \Omega\right) \\
&>C \omega\left(f(z), \bigcup_{j \in J}\left(l_{j} \cap \frac{1}{2} R_{j}^{m}\right), \Omega_{1}\right) .
\end{aligned}
$$

By (5.2), the left-hand side of the above inequality is small. Hence (6.11) follows from Lemma 4.2 .

Since $\sum\left|I_{i}\right|+\sum\left|p\left(\operatorname{bad} l_{i}\right)\right| \ll 1,(6.11)$ implies

$$
\mid \bigcup\left\{\left(a_{i}, b_{i}\right) \text { of Case III satisfying properties (i)-(iii) }\right\} \mid \geq \frac{15}{31}
$$

where (i)-(iii) are as follows:

(i) $\frac{1}{2} R_{i}^{m} \cap \partial \Omega=\emptyset$;

(ii) $l_{i}$ is good;

(iii) $\mid \bigcup\left\{I_{j}\right.$ located under $\left.\frac{1}{2} R_{i}^{m}\right\}|+| \bigcup\left\{p\left(\right.\right.$ bad $l_{j}$ located under $\left.\left.\frac{1}{2} R_{i}^{m}\right)\right\} \mid$ $<\frac{1}{15}\left|p\left(\frac{1}{2} R_{i}^{m}\right)\right|$. For $\left(a_{i}, b_{i}\right)$ satisfying (i)-(iii), let $J_{i}$ be the top of $\frac{1}{2} R_{i}^{m}$ and let $Q_{i}$ be a rectangle with top $J_{i}$ containing the subarc of $\Gamma$ located under $J_{i}$. We say that $\left(a_{i}, b_{i}\right)$ is good if there exist disjoint balls $B_{k}^{i}$ in $Q_{i}$ such that

(i) $\operatorname{center}\left(B_{k}^{i}\right) \in f(A)$;

(ii) $B_{k}^{i} \cap Q_{j}=\emptyset$ for $j \neq i$;

(iii) $\sum_{k} \operatorname{rad}\left(B_{k}^{i}\right) \geq \frac{1}{12}\left|J_{i}\right|$. 

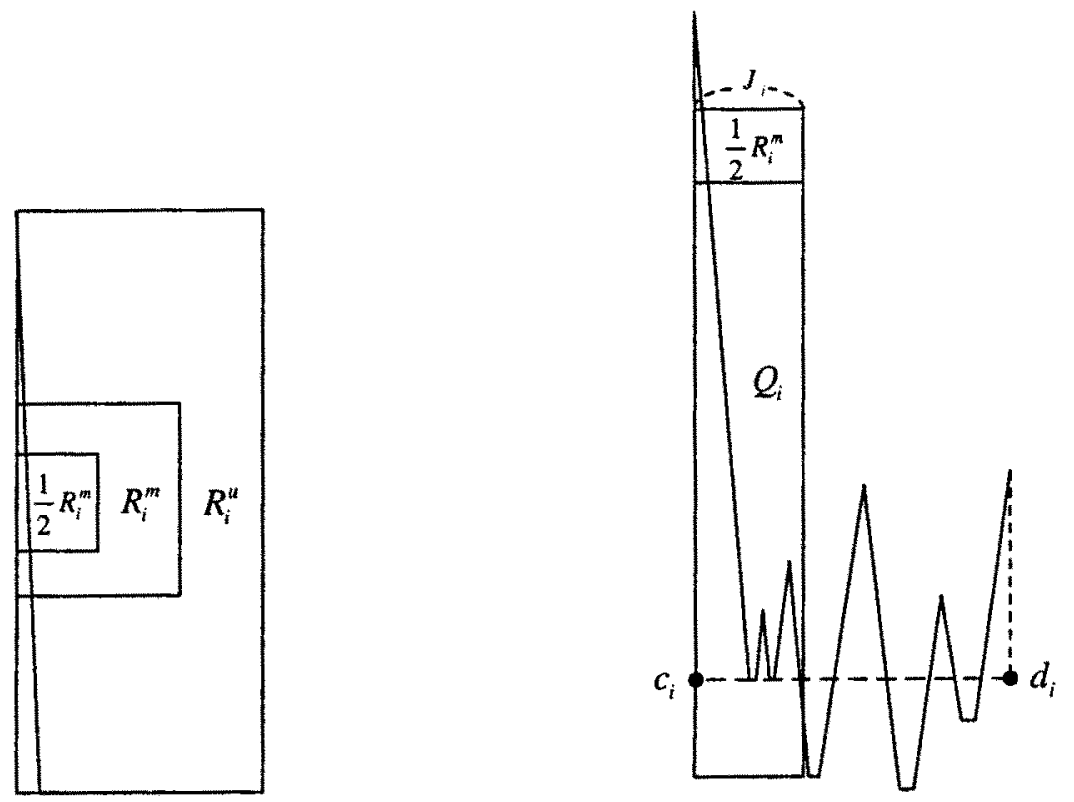

Figure 11. $\frac{1}{2} R_{i}^{m}, J_{i}$ and $Q_{i}$ of Case III.

If

$$
\sum\left|\operatorname{good}\left(a_{i}, b_{i}\right)\right| \geq \frac{12}{31},
$$

then $\left\{B_{k}^{i}\right\}_{i, k}$ is disjoint and

$$
\sum_{i, k} \operatorname{rad}\left(B_{k}^{i}\right) \geq \frac{1}{12} \cdot \frac{700}{3} \cdot \frac{12}{31}>(1.01) \cdot 6 \geq(1.01) r_{0}
$$

Hence it suffices to consider the case when

$$
\sum\left|\operatorname{good}\left(a_{i}, b_{i}\right)\right|<\frac{12}{31},
$$

which is equivalent to

$$
\sum\left|\operatorname{bad}\left(a_{i}, b_{i}\right)\right|>\frac{3}{31} .
$$

Under the assumption (6.12), we show that $\omega\left(f(z), \beta \backslash f\left(A^{\prime}\right), \Omega\right)$ is bounded below. This contradicts (5.2) and completes the proof of Theorem 2.

Now, assume (6.12). For bad $\left(a_{i}, b_{i}\right)$, let $\beta_{i}$ be a subarc of $\beta$ connecting a point in $I_{i}$ and the highest point in $\partial Q_{i} \cap \beta$. This point cannot be on the top edge of $Q_{i}$ or on the side below $\Gamma$, since $\frac{1}{2} R_{i}^{m} \cap \partial \Omega=\emptyset$ and $l_{i}$ is good. Also, $\beta_{i}$ can leave and reenter $Q_{i}$. Let $x_{i}$ be the middle point of $\frac{1}{2} R_{i}^{m}$. (See Figure 12.) We shall show 


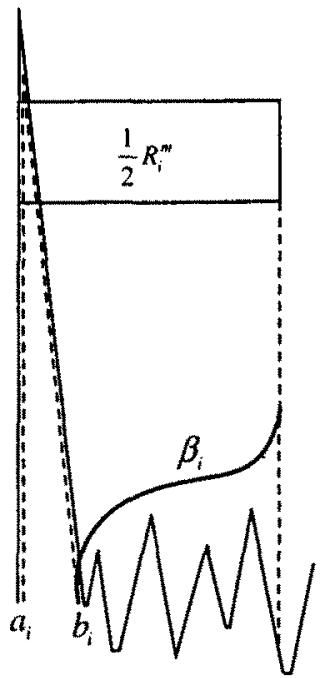

Figure 12. $\beta_{i}$ of Case III.

that for some $c^{\prime}>0$,

$$
\omega\left(x_{i}, \beta_{i} \backslash f\left(A^{\prime}\right), \Omega\right)>c^{\prime} .
$$

If (6.13) holds, then by (6.12) and Lemma 4.2 ,

$$
\omega\left(f(z), \beta \backslash f\left(A^{\prime}\right), \Omega\right) \gtrsim \sum_{\operatorname{bad}\left(a_{i}, b_{i}\right)} \omega\left(f(z), \partial B\left(x_{i}, \frac{1}{4}\left|J_{i}\right|\right), \Omega_{1}^{\prime \prime}\right) \cdot \omega\left(x_{i}, \beta_{i} \backslash f\left(A^{\prime}\right), \Omega\right)>c^{\prime \prime}
$$

for some $c^{\prime \prime}>0$, where

$$
\Omega_{1}^{\prime \prime}=\left(\Omega_{1} \bigcup\left(\bigcup_{\substack{\text { bad } \\\left(a_{i}, b_{i}\right)}} \frac{1}{2} R_{i}^{m}\right)\right) \backslash\left(\bigcup_{\substack{\text { bad } \\\left(a_{i}, b_{i}\right)}} B\left(x_{i}, \frac{1}{4}\left|J_{i}\right|\right)\right) .
$$

(6.14) contradicts (5.2), as desired.

Hence it suffices to prove (6.13). We fix a bad $\left(a_{i_{0}}, b_{i_{0}}\right)$ and prove (6.13) in three steps.

\section{Step 1. Construction of $\Omega_{3}$}

Let $\left\{Q_{j}: j \in J\right\}$ be the set of $Q_{i}$ 's located under $J_{i_{0}}$ and let

$$
\Omega^{\prime \prime \prime}=\Omega_{1} \bigcup\left(\bigcup_{j \in J} Q_{j}\right)
$$



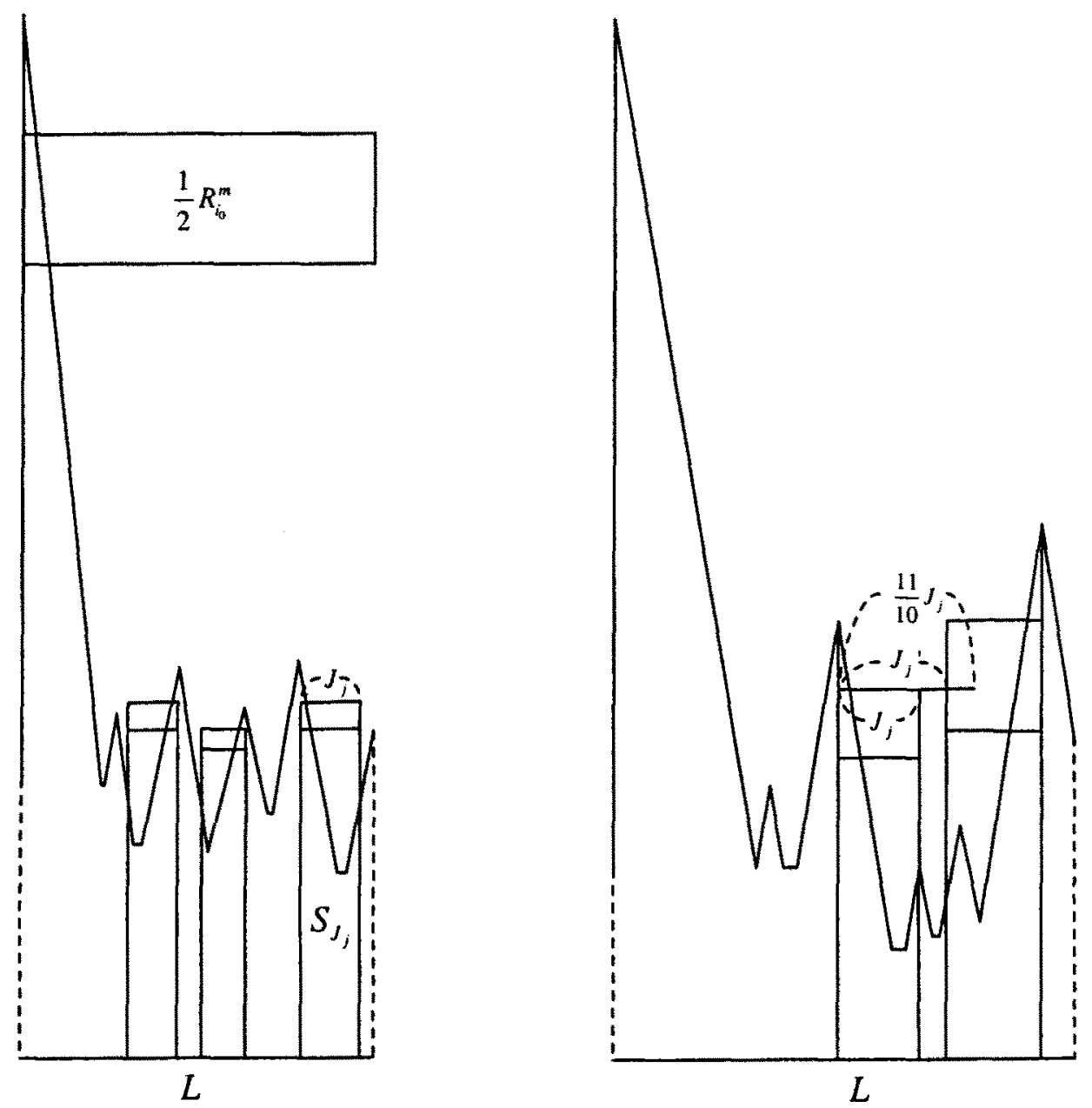

Figure 13. $S_{J_{j}}$ and $J_{j}^{\prime}$ of Step 1.

For the top $J_{j}$ of $Q_{j}$, let $\frac{11}{10} J_{j}$ denote the line segment with one endpoint at endpoint $\left(J_{j}\right) \cap \Omega_{1}$ such that $J_{j} \subset \frac{11}{10} J_{j}$ and $\left|\frac{11}{10} J_{j}\right|=\frac{11}{10}\left|J_{j}\right|$.

If $\frac{11}{10} J_{j}$ intersects $\partial \Omega^{\prime \prime \prime}$, then extend $J_{j}$ until it meets $\partial \Omega^{\prime \prime \prime}$ and let $J_{j}^{\prime}$ denote the extended line segment. (See Figure 13.) Define

$$
\Omega_{3}=\Omega^{\prime \prime \prime} \bigcup\left(\bigcup S_{J_{j}^{\prime}}\right) \text {, }
$$

where $S_{J_{j}^{\prime}}$ is the rectangle with top $J_{j}^{\prime}$ and bottom on $L$. Then $\Omega_{3}$ is a chord-arc domain; and for $K_{j}=J_{j}^{\prime} \backslash J_{j}$,

$$
\sum_{j \in J}\left|K_{j} \cap \partial \Omega_{3}\right| \leq \frac{1}{10}\left|J_{i_{0}}\right|
$$




\section{Step 2. Construction of $\Omega_{4}$}

For $q \in f\left(A^{\prime}\right)$ located between $J_{i_{0}}$ and $\partial \Omega_{3}$, let $B_{q}$ be the ball adjacent to $\partial\left(Q_{i_{0}} \backslash \Omega_{3}\right)$ with center at $q$. We also let $T_{q}$ be the horizontal diameter of $B_{q}$.

If

$$
\left|\bigcup_{q} p\left(T_{q}\right)\right|>\frac{1}{3}\left|J_{i_{0}}\right|
$$

then by Besicovitch's covering lemma, there exists a disjoint collection $\left\{p\left(T_{q_{k}}\right)\right\}$ such that

$$
\left|\bigcup_{k} p\left(T_{q_{k}}\right)\right|>\frac{1}{6}\left|J_{i_{0}}\right| .
$$

Observe that $\left\{B_{q_{k}}\right\}$ is disjoint and satisfies the conditions (i)-(iii) of the definition of good $\left(a_{i}, b_{i}\right)$. This contradicts the fact that $\left(a_{i_{0}}, b_{i_{0}}\right)$ is bad. Hence we obtain

$$
\left|\bigcup_{q} p\left(T_{q}\right)\right| \leq \frac{1}{3}\left|J_{i_{0}}\right| .
$$

Let

$$
\Omega^{\prime \prime \prime \prime}=\left(Q_{i_{0}} \backslash \Omega_{3}\right) \backslash\left(\bigcup_{q} S_{T_{q}}\right),
$$

where $S_{T_{q}}$ is the rectangle with top $T_{q}$ and bottom on $L$. (See Figure 14.) If $2 T_{q}$ intersects $\partial \Omega^{\prime \prime \prime}$, then extend $T_{q}$ until it meets $\partial \Omega^{\prime \prime \prime}$ and let $T_{q}^{t}$ denote the extended line segment. Define

$$
\Omega_{4}=\Omega^{\prime \prime \prime} \backslash\left(\bigcup S_{T_{q}^{\prime}}\right)
$$

then $\Omega_{4}$ is a chord-arc domain, and

$$
\Omega_{4} \cap f\left(A^{\prime}\right)=\emptyset
$$

$\Omega_{3}$ and $\Omega_{4}$ are disjoint chord-arc domains satisfying

$$
\left|p\left(\partial \Omega_{3} \cap \partial \Omega_{4}\right)\right| \geq \frac{1}{3}\left|J_{i_{0}}\right| .
$$

Step 3. Contradicting the smallness property of $\omega\left(x_{i_{0}}, \beta_{i_{0}} \backslash f\left(A^{\prime}\right), \Omega\right)$

For good $l_{i}$ located under $J_{i_{0}}$, let $l_{i}^{\prime}$ be the line segment in $\Omega_{1}$ such that $l_{i}^{t}$ is parallel to $l_{i}, p\left(l_{i}^{\prime}\right)=p\left(l_{i}\right)$ and $\operatorname{dist}\left(l_{i}, l_{i}^{\prime}\right)=c\left|a_{i}-b_{i}\right|$ for the constant $c$ of (6.6). Then

$$
\text { the region between } l_{i}^{t} \text { and } L \text { is contained in } \Omega \text {. }
$$

Also, for $J_{j}$ located under $J_{i_{0}}$,

$$
\frac{1}{2} R_{j}^{m} \subset \Omega
$$



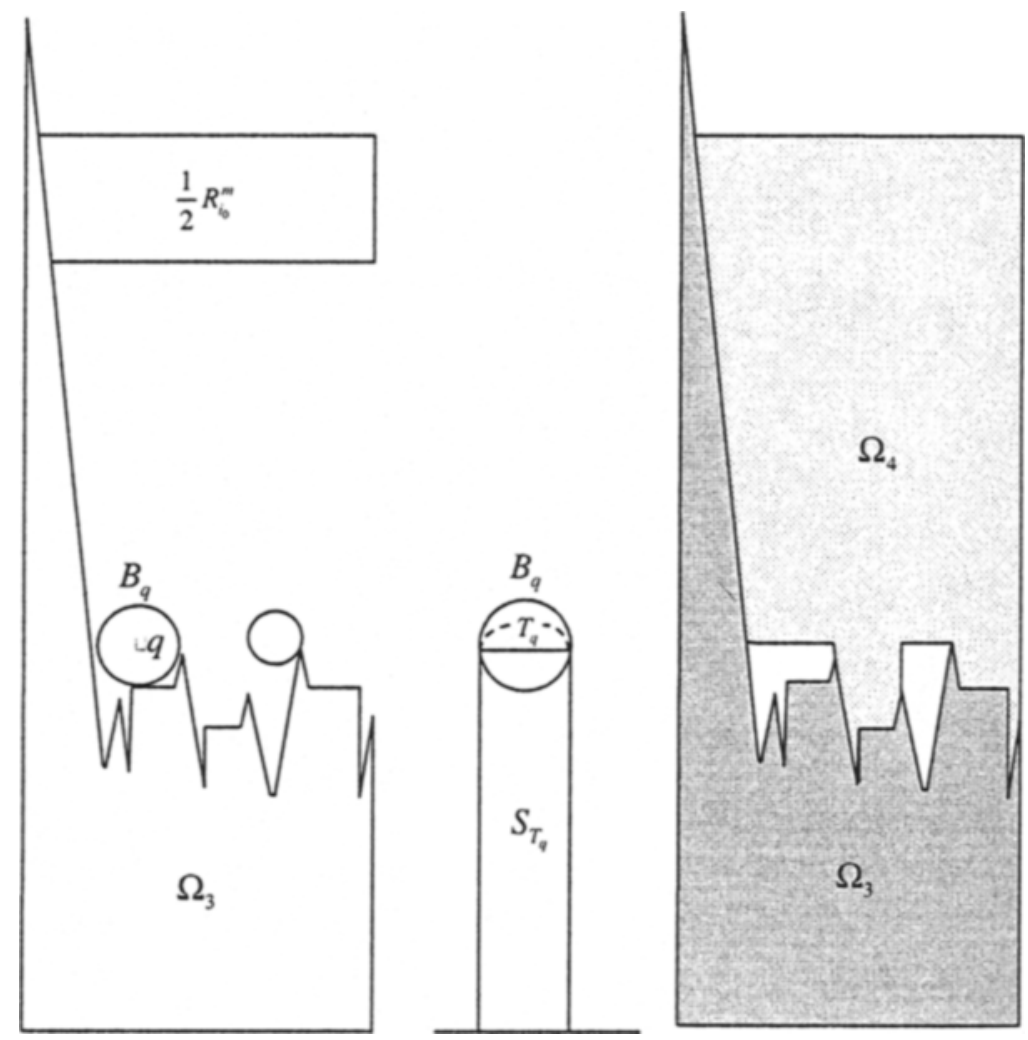

Figure 14. $\Omega_{3}$ of Step 1 and $\Omega_{4}$ of Step 2 .

If $\beta_{i_{0}}$ is replaced by an arc on $\partial \Omega_{4}$ which has the same endpoints and separates a point of $\beta_{i_{0}}$ from $x_{i_{0}}$, then by (6.15), (6.16) and (6.17),

$$
\omega\left(x_{i_{0}}, \beta_{i_{0}} \backslash f\left(A^{\prime}\right), \Omega\right) \gtrsim \omega\left(x_{i_{o}},\left(\bigcup J_{j}\right) \bigcup\left(\bigcup \operatorname{good} l_{i}\right), \Omega_{4}\right) .
$$

But

$$
\begin{aligned}
\left|p\left(\partial \Omega_{4} \bigcap\left(\left(\bigcup J_{j}\right) \bigcup\left(\bigcup \operatorname{good} l_{i}\right)\right)\right)\right| & >\left|J_{i_{0}}\right|-\left|\bigcup_{q} p\left(2 T_{q}\right)\right|-\sum_{j}\left|K_{j} \cap \partial \Omega_{3}\right| \\
-\mid \bigcup\left\{I_{i} \text { located under } J_{i_{0}}\right\} \mid & -\mid p\left(\bigcup\left\{\operatorname{bad} l_{i} \text { located under } J_{i_{0}}\right\}\right) \mid \\
& >\left(1-\frac{2}{3}-\frac{1}{10}-\frac{1}{15}\right)\left|J_{i_{0}}\right|=\frac{1}{6}\left|J_{i_{0}}\right| .
\end{aligned}
$$

The above inequality and Lemma 4.2 show that the right side of $(6.18)$ is bounded below. Hence we obtain (6.13) for bad $\left(a_{i}, b_{i}\right)$, and the proof of Theorem 2 is complete. 
Recall that we assumed $r=\frac{1}{5} r_{0}$ in Section 5. In case $r<\frac{1}{5} r_{0}$, construct $L$ satisfying $|L| \geq \frac{5}{6} r$ and make the Lipschitz constant of $\Gamma$ sufficiently greater than 98000. Then we can construct disjoint balls $B_{k}$ with centers in $f(A)$ such that

$$
\sum \operatorname{rad}\left(B_{k}\right)>(1.01) \cdot \frac{r_{0}}{r} \cdot \frac{6}{5} \cdot|L| \geq(1.01) r_{0} .
$$

But since $r \approx r_{0}$, the Lipschitz constant of $\Gamma$ can be bounded above. Also, every constant in the proof depends only on the Lipschitz constant of $\Gamma$. Hence the proof works for every $r \approx r_{0}$, if we take the Lipschitz constant of $\Gamma$ sufficiently large.

Acknowledgment. The author thanks Prof. John Garnett at University of California, Los Angeles, for his consistent advice and encouragement.

\section{REFERENCES}

[BiJo 90] C. Bishop and P. Jones, Harmonic measure and arclength, Ann. Math. 132 (1990), 511 547.

[BiJo 94] C. Bishop and P. Jones, Harmonic measure, $L^{2}$ estimates and the Schwarzian derivative, J. Analyse Math. 62 (1994), 77-113.

[Bis] C. Bishop, Harmonic measure and Hausdorff dimension, in Linear and Complex Analysis Problem Book 3, Part 2, Springer-Verlag, Berlin, 1994, pp. 385-387.

[Bis 91] C. Bishop, Some questions concerning Harmonic measure, in Partial Differential Equations with Minimal Smoothness and Applications (B. Dahlberg, E. Fabes, R. Fefferman, D. Jerison, C. Kenig and J. Pipher, eds.), Springer-Verlag, Berlin, 1991.

[GaMa] J. Garnett and D. Marshall, Harmonic Measure, Cambridge University Press, to appear.

[JeKe 82] D. Jerison and C. Kenig, Hardy spaces, $A_{\infty}$, and singular integrals on chord-arc domains Math. Scand. 50 (1982), 221-247.

[Mak 85] N. Makarov, On the distortion of boundary sets under conformal mappings, Proc, London Math. Soc. 51 (1985), 369-384.

[Mat 95] P. Mattila, Geometry of Sets and Measures in Euclidean Spaces, Cambridge University Press, 1995.

[McM 70] J. E. McMillan, On the boundary correspondence under conformal mapping, Duke Math. J. 37 (1970), 725-739.

[Øks 81] B. Oksendal, Brownian motion and sets of harmonic measure zero, Pacific J. Math. 95 (1981), 179-192.

[Pom 86] Ch. Pommerenke, On conformal mapping and linear measure, J. Analyse Math. 46 (1986), 231-238.

[Pom 91] Ch. Pommerenke, Boundary Behavior of Conformal Maps, Springer-Verlag, Berlin, 1991.

\section{Sunhi Choi}

DEPARTMENT OF MATHEMATICS

UNIVERSITY OF CALIFORNIA

LOS ANGELES, CA 90095-1555, USA

email: schoi@math.ucla.edu

(Received December 16, 2002 and in revised form August 25, 2003) 